\title{
Momentum dissipation and holographic transport without self-duality
}

\author{
Jian-Pin Wu $\mathbf{u}^{1,2, a}$, Xiao-Mei Kuang ${ }^{1, b}$, Guoyang $\mathbf{F u}^{2, c}$ \\ ${ }^{1}$ Center for Gravitation and Cosmology, College of Physical Science and Technology, Yangzhou University, Yangzhou 225009, China \\ ${ }^{2}$ Department of Physics, School of Mathematics and Physics, Bohai University, Jinzhou 121013, China
}

Received: 4 November 2017 / Accepted: 24 July 2018 / Published online: 1 August 2018

(C) The Author(s) 2018

\begin{abstract}
We explore the response of the momentum dissipation introduced by spatial linear axionic fields in a holographic model without self-duality, which is broke by Weyl tensor coupling to Maxwell field. It is found that for the positive Weyl coupling parameter $\gamma>0$, the momentum dissipation, characterized by parameter $\hat{\alpha}$, drives an incoherent metallic state with a peak at low frequency into another incoherent metallic phase with a dip. While for $\gamma<0$, an oppositive scenario is observed. Another interesting feature in our model is that for some observables including the DC conductivity, diffusion constant and susceptibility, there exists a certain value of $\hat{\alpha}$, for which these observables are independent of $\gamma$. Finally, the electromagnetic (EM) duality is also studied and there is also a specific value of $\hat{\alpha}$, for which the particle-vortex duality related by the change of the sign of $\gamma$ in the boundary theory holds better than for other values of $\hat{\alpha}$.
\end{abstract}

\section{Introduction}

The transport properties, such as the electrical conductivity, heat conductivity and thermoelectric transport, are important features of real materials. For the weakly coupled systems, the frequency dependent conductivity exhibits Drudelike peak at low frequency. Their collective dynamics is well described by the quantum Boltzmann theory of the quasi-particles with long-lived excitations [1]. While for the strongly coupled systems, the picture of the quasi-particle is absent and the Boltzmann theory is usually invalid. ${ }^{1}$

\footnotetext{
1 When quasi-particle excitations are only weakly broken, the perturbative method in the Boltzmann framework is developed to deal with such systems, see for example, [1-4].

a e-mail: jianpinwu@mail.bnu.edu.cn

b e-mail: xmeikuang@gmail.com

c e-mail: FuguoyangEDU@163.com
}

The anti-de Sitter/conformal field theory (AdS/CFT) correspondence [5-9] provides a powerful tool and novel mechanism to study the transport of the strongly coupled systems. One of the long-standing important issues in strongly coupling systems is the quantum critical (QC) dynamics described by CFT [10] (also refer to [1,4,11-16]). A controlled manner in traditional field theory is absent in studying the QC physics at finite temperature. Also the numerical simulations suffer from the "sign" problem and usually fail. Here the power of holography is evident.

More recently, remarkable progresses have been made in the study of the transport properties of QC physics by holography [17-22]. They introduced an extra four-derivative interaction, the Weyl tensor $C_{\mu \nu \rho \sigma}$, coupled to Maxwell field, in the Schwarzschild-AdS (SS-AdS) black brane, which is dual to a neutral plasma at finite temperature, and then study the transport behavior of the QC physics. ${ }^{2}$ Since the breakdown of the electromagnetic (EM) self-duality, a frequency dependent optical conductivity is observed in this neutral plasma $^{3}$ [17]. In particular, the conductivity at low frequency displays a peak for $\gamma>0$, which resembles the particle excitation described by the Boltzmann theory [17]. While for $\gamma<0$, it exhibits a dip and is similar to the vortex case [17]. Here $\gamma$ is the parameter controlling the coupling strength of the Weyl term. It also provides a possible route to access the CFT of the superfluid-insulator quantum critical point (QCP) described by the boson Hubbard model though there is still a degree of freedom of the sign of $\gamma^{4}$ [25]. Also they found that a particle-vortex duality in the dual bound-

\footnotetext{
2 The transport of the Maxwell-Weyl system in a perturbative higherderivative neutral background has also been studied in [23].

${ }^{3}$ Note that due to the EM self-duality, the optical conductivity is frequency independent in the neutral system dual to the standard Maxwell theory in four dimensional SS-AdS bulk spacetime [24].

${ }^{4}$ We provide a brief introduction on this problem in Sect. 4 . For the more details, please see [25].
} 
ary field theory, which is related by the change of the sign of $\gamma$ [17-22]. Furthermore, based on this framework [17], some important results are achieved [18-22]. For instance, by combining high precision quantum Monte Carlo (QMC) simulations with the results from the boundary CFT dual to the Maxwell-Weyl system in SS-AdS geometry, a quantitative description of the transports of QC physics without quasi-particle excitation is built [20,22], which is experimentally testable.

In this paper, we intend to implement the momentum dissipation into the Maxwell-Weyl system studied in [17-22] and investigate its response. There are many ways to implement the momentum dissipation in holographic manner, see for example [26-33], and many interesting results have been obtained, for example [34-44]. Here we adopt a simple way proposed in [33], where the momentum dissipation is implemented by a pair of massless field, $\Phi_{I}$ with $I=1,2$, which are spatial linear dependent in the bulk. It is also referred as "mean-field disordered" [45] due to the homogeneous background geometry. Note that $\Phi_{I}$ correspond to turning on spatial linear sources in the dual boundary theory, i.e.,

$\phi_{I}^{(0)} \propto \alpha x_{I}$,

with $\alpha$ being constant. This nonuniform source means that a dimensionful parameter, i.e., $\alpha$, is introduced into the dual boundary theory, and so the physics we study is that away from QCP. We hope that our present model provides wider route to address whether the excitation of the CFT of the superfluid-insulator QCP described by the boson Hubbard model is particle-like or vortex-like, and also toward the problem which sign of $\gamma$ is the correct description of this CFT. In addition, the proximity effect in QCP, which also alters some observables such as the optical conductivity, is also important and has been explored in $[1,4,10-16,46]$. Our present work will also provide some insight into the transport properties away from QCP in holographic framework.

Our paper is organized as follows. We begin with a review of the holographic framework without EM self-duality in Sect. 2. We then introduce a neutral axionic theory, which is responsible for the momentum dissipation in Sect. 3. The optical conductivity of the boundary field theory dual to the Maxwell-Weyl system in the neutral axionic geometry is studied in Sect. 4. We mainly focus on the role of the momentum dissipation plays in the transport properties in our present model. We also study the diffusion constant and susceptibility of the dual boundary field theory in Sect. 7. In Sect. 6, we discuss the EM duality. We conclude with a brief discussion and some open questions in Sect. 7. In Appendix A, we discuss the constraints imposing on the Weyl coupling parameter $\gamma$ in the neutral axionic geometry due to the causality and the instabilities.

\section{Holographic framework without EM self-duality}

The optical conductivity in the neutral plasma, which is dual to the standard Maxwell theory in four dimensional AdS spacetimes, does not depend on the frequency due to the EM self-duality [24]. Thus, in order to have a frequency dependent optical conductivity, we need to break the EM self-duality. A simple way is to introduce the Weyl tensor $C_{\mu \nu \rho \sigma}$ coupling to gauge field as $[17-23,47]$

$S_{1}=\frac{1}{g_{F}^{2}} \int d^{4} x \sqrt{-g}\left(-\frac{1}{4} F_{\mu \nu} F^{\mu \nu}+\gamma C_{\mu \nu \rho \sigma} F^{\mu \nu} F^{\rho \sigma}\right)$,

where $F=d A$ is the curvature of gauge field $A$ and $g_{F}^{2}$ is an effective dimensionless gauge coupling, which shall be set $g_{F}=1$ in the numerical calculation. In this theory, the crucial dimensionless coupling parameter, $\gamma$, controls the coupling strength of the Maxwell-Weyl term. The Weyl term is a specific combination of some four-derivative interaction term [17], which can be expected to emerge as quantum corrections in the low energy effective action in string theory context $[48,49]$.

For convenience, we write down the action (2) in a general form [17] (also see [18-22])

$S_{A}=\int d^{4} x \sqrt{-g}\left(-\frac{1}{8 g_{F}^{2}} F_{\mu \nu} X^{\mu \nu \rho \sigma} F_{\rho \sigma}\right)$,

where a new tensor $X$ is introduced as

$X_{\mu \nu}^{\rho \sigma}=I_{\mu \nu}^{\rho \sigma}-8 \gamma C_{\mu \nu}^{\rho \sigma}$,

with an identity matrix $I_{\mu \nu}{ }^{\rho \sigma}=\delta_{\mu}{ }^{\rho} \delta_{\nu}{ }^{\sigma}-\delta_{\mu}{ }^{\sigma} \delta_{\nu}{ }^{\rho}$ acting on the two-forms. It is easy to find that the tensor $X$ possess the symmetries

$X_{\mu \nu \rho \sigma}=X_{[\mu \nu][\rho \sigma]}=X_{\rho \sigma \mu \nu}$.

When $X_{\mu \nu}^{\rho \sigma}=I_{\mu \nu}^{\rho \sigma}$, the theory with (2) reduces to the standard Maxwell theory. Moreover, in this case, the equation of motion for the action (3) is

$\nabla_{\nu}\left(X^{\mu \nu \rho \sigma} F_{\rho \sigma}\right)=0$.

It was addressed in [17] that the Weyl term breaks down the EM self-duality, however, one can still construct the dual EM theory for the gauge theory (2). Thus, here we directly write down the corresponding dual EM theory

$S_{B}=\int d^{4} x \sqrt{-g}\left(-\frac{1}{8 \hat{g}_{F}} G_{\mu \nu} \widehat{X}^{\mu \nu \rho \sigma} G_{\rho \sigma}\right)$,

where $\hat{g}_{F}^{2} \equiv 1 / g_{F}^{2}$ and $G_{\mu \nu} \equiv \partial_{\mu} B_{v}-\partial_{\nu} B_{\mu}$. In addition, the tensor $\widehat{X}$ is defined by

$\widehat{X}_{\mu \nu}^{\rho \sigma}=-\frac{1}{4} \varepsilon_{\mu \nu}^{\alpha \beta}\left(X^{-1}\right)_{\alpha \beta}^{\gamma \lambda} \varepsilon_{\gamma \lambda}^{\rho \sigma}$, 
where $\varepsilon_{\mu \nu \rho \sigma}$ is volume element and $X^{-1}$ is defined by

$\frac{1}{2}\left(X^{-1}\right)_{\mu \nu}^{\rho \sigma} X_{\rho \sigma}^{\alpha \beta} \equiv I_{\mu \nu}^{\alpha \beta}$

Then we obtain the equation of motion for the dual theory (7)

$\nabla_{\nu}\left(\widehat{X}^{\mu \nu \rho \sigma} G_{\rho \sigma}\right)=0$.

For the standard four-dimensional Maxwell theory, $\widehat{X}_{\mu \nu}^{\rho \sigma}$ $=I_{\mu \nu}^{\rho \sigma}$ and therefore the theory (3) and (7) are identical, which means that the Maxwell theory is self-dual. When the Weyl term is introduced, we find that for small $\gamma$,

$$
\begin{aligned}
\left(X^{-1}\right)_{\mu \nu}^{\rho \sigma} & =I_{\mu \nu}^{\rho \sigma}+8 \gamma C_{\mu \nu}^{\rho \sigma}+\mathcal{O}\left(\gamma^{2}\right), \\
\widehat{X}_{\mu \nu}^{\rho \sigma} & =\left(X^{-1}\right)_{\mu \nu}^{\rho \sigma}+\mathcal{O}\left(\gamma^{2}\right)
\end{aligned}
$$

which implies that the self-dual is violated for the theory (2), and there is a duality between the actions (3) and (7) with the change of the sign of $\gamma$.

\section{A neutral axionic theory}

We intend to implement the momentum dissipation in the Maxwell-Weyl system and study its response. The simplest way is to introduce a pair of spatial linear dependent axionic fields into the action of the Einstein gravity [33],

$S_{0}=\frac{1}{2 \kappa^{2}} \int d^{4} x \sqrt{-g}\left(R+6-\frac{1}{2} \sum_{I=x, y}\left(\partial \phi_{I}\right)^{2}\right)$,

where $\phi_{I}=\alpha x_{I}$ with $I=x, y$ and $\alpha$ being a constant. The negative cosmological constant $\Lambda=-6$ in the action supports an asymptotically AdS spacetimes. ${ }^{5}$ When the momentum dissipation is weak, the standard Maxwell theory with the action (13) describes coherent metallic behavior of the finite density dual boundary theory, however, once the momentum dissipation is strong, we can have an incoherent metal [33,50-52].

We shall study the system described by the actions $S_{1}$ in Eq. (2) and $S_{0}$ in Eq. (13). However, it is difficult to numerically solve the equations of motions for the EinsteinMaxwell-axion-Weyl (EMA-Weyl) theory [Eqs. (2) and (13)] because they are a set of third order nonlinear differential equations. Luckily, up to the first order of the Weyl coupling parameter $\gamma$, the analytical background solutions has been constructed [53-59] and the DC conductivity was studied in [59]. However, it is still difficult to obtain the frequency dependent conductivity. As the first step, here we shall follow the strategy in [17-23] and study the transports of the

\footnotetext{
5 Here, without loss of generality we have set the AdS radius $L=1$ for simplicity.
}

Maxwell-Weyl system (2) in the neutral plasma dual to the Einstein-axions (EA) theory (13).

The neutral black brane solution of the EA action (13) can be written down as [33]

$d s^{2}=\frac{1}{u^{2}}\left(-f(u) d t^{2}+\frac{1}{f(u)} d u^{2}+d x^{2}+d y^{2}\right)$,

where

$f(u)=(1-u) p(u), \quad p(u)=\left(1-\frac{\alpha^{2}}{2}\right) u^{2}+u+1$.

$u=0$ is the asymptotically AdS boundary while the horizon locates at $u=1$. The Hawking temperature can be expressed as

$T=\frac{p(1)}{4 \pi}=\frac{3-\frac{\alpha^{2}}{2}}{4 \pi}$.

And the energy density $\epsilon$, pressure $p$ and entropy density $s$ of the dual boundary theory can be calculated as [33]

$\epsilon=2\left(1-\frac{\alpha^{2}}{2}\right), \quad p=1+\frac{\alpha^{2}}{2}, \quad s=4 \pi$.

Since the black brane solution (14) and (15) is only parameterized by one scaling-invariant parameter $\hat{\alpha}=\alpha / 4 \pi T$, for later convenience, we reexpress the function $p(u)$ as

$p(u)=\frac{\sqrt{1+6 \hat{\alpha}^{2}}-2 \hat{\alpha}^{2}-1}{\hat{\alpha}^{2}} u^{2}+u+1$.

Before processing, we would give some comments on the solution. First, it is easy to see from (16) that at zero temperature $(\alpha=\sqrt{6})$, the IR geometry is $\operatorname{AdS}_{2} \times \mathbb{R}_{2}$, which is similar to the Reissner-Nordström-AdS (RN-AdS) black brane. Second, there is special value $\alpha=\sqrt{2}$ where the energy density $\epsilon$ vanishes. In this case, there is selfduality in Maxwell equations and the AC heat conductivity is frequency-independent [52]. Third, the axionic fields $\phi_{I}$ in bulk is dual to turning on linearly spatial dependent sources in the dual boundary theory. Such sources result in the momentum dissipation, which is controlled by the parameter $\hat{\alpha}$. Meanwhile, as pointed out in the introduction, $\Phi_{I}$ introduce the nonuniform sources with a dimensional parameter $\alpha$ in the dual boundary theory such that the system we are studying is away from QCP.

\section{Optical conductivity}

In this paper, we mainly study the electrical optical conductivity at zero momentum, which is a simple but important transport behavior. The other transport properties, such as the thermal conductivity, the optical conductivity at finite momentum, will be studied elsewhere. 
To this end, we turn on the perturbations of the gauge field and decompose it in the Fourier space as

$A_{\mu}(t, x, y, u)=\int \frac{d^{3} q}{(2 \pi)^{3}} e^{i \mathbf{q} \cdot \mathbf{x}} A_{\mu}(u, \mathbf{q})$,

where $\mathbf{q} \cdot \mathbf{x}=-\omega t+q^{x} x+q^{y} y$. Without loss of generality, we set $\mathbf{q}^{\mu}=(\omega, q, 0)$ and choose the gauge fixed as $A_{u}(u, \mathbf{q})=$ 0 . In this section, we shall study the optical conductivity at zero momentum, so we set $q=0$. For $q=0$, all the perturbative equations of the gauge field [Eqs. (A3)-(A6) in Appendix A] decouple. Therefore, we only need to solve Eqs. (A5) or (A6) to obtain the optical conductivity. Due to the symmetry between $x$ and $y$ directions, we will focus on the conductivity in $y$ direction, which read as

$\sigma(\omega)=\frac{\partial_{u} A_{y}(u, \hat{\omega}, \hat{q}=0)}{i \omega A_{y}(u, \hat{\omega}, \hat{q}=0)}$.

In addition, through this paper, we use the dimensionless frequency and momentum, which are defined as

$$
\begin{aligned}
\hat{\omega} & \equiv \frac{\omega}{4 \pi T}=\frac{\omega}{\mathfrak{p}}, \quad \hat{q} \equiv \frac{q}{4 \pi T}=\frac{q}{\mathfrak{p}}, \quad \mathfrak{p} \\
& \equiv p(1)=4 \pi T .
\end{aligned}
$$

\subsection{Optical conductivity}

Before processing, we briefly review the problem about the boson Hubbard model, which was addressed in [25]. In the insulating phase of the model, it is the excitation of the particle and hole, so we can infer that the conductivity at low frequency should exhibit a peak when we approach the QCP from the insulator side. When we approach the QCP from the superfluid side which is described by the excitation of the vortices, then the conductivity at low frequency should be a dip. However, which of the two qualitatively distinct results is the real physics still needs to be further disclosed.

The Maxwell-Weyl system in the SS-AdS geometry provides a possible description for the CFT of the boson Hubbard model in holographic framework [17-22], though one still have a degree of freedom for the sign of $\gamma$. In details, the optical conductivity of the boundary field theory dual to SS-AdS geometry has been explored. It was found that for $\gamma>0$, it exhibits a peak at low frequency, ${ }^{6}$ which qualitatively resembles the Boltzmann transport of particles. While for $\gamma<0$, a dip appears, which is similar to the excitation of vortices. Somehow, it provides a possible route to resolve whether the excitation of the CFT of the superfluid-insulator QCP described by the boson Hubbard model is particle-like or vortex-like.

\footnotetext{
${ }^{6}$ There is a deviation from the standard Drude formula for $\gamma \in \mathcal{S}_{0}$ with $\mathcal{S}_{0}:=[-1 / 12,1 / 12]$ shown in the Appendix A. We shall illustrate this point below.
}

Here, we hope that implementing the momentum dissipation, which is also equivalent to the "mean-field disordered" effect [45], will provide more clues in addressing this problem.

The top plots of Fig. 1 show the real and imaginary part of the optical conductivity $\sigma(\hat{\omega})$ for $\gamma=1 / 12$ and different $\hat{\alpha}$. We observe that at small $\hat{\alpha}$, a peak displays in the low frequency optical conductivity, and gradually degrades as $\hat{\alpha}$ increases, eventually becomes a dip. It implies that the disorder effect introduced by the axions drives the dual CFT with positive $\gamma$ into the incoherent metallic phase with a dip. While for $\gamma<0$, an oppositive scenario is found (see the bottom plots of Fig. 1). That is to say, as $\hat{\alpha}$ increases, the dip in $\sigma(\hat{\omega})$ at low frequency gradually upgrades and eventually develops into a peak. It indicates that if the dual CFT is with negative $\gamma$, then the disorder drives it into the metallic phase characterized by a peak. Though the present model still cannot give a definite answer to which sign of $\gamma$ is the realistic description of the CFT of boson Hubbard model, it indeed provide a route toward this problem.

It is notable that in this holographic framework, one can introduce different types of disorder, for example the Qlattice $[30,31]$, into the Maxwell-Weyl system to see how the universal results will be presented. We shall address this problem in near future. Next, we present more details on the optical conductivity of our present model, in particular its low frequency behavior.

\subsection{The low frequency behavior of the optical conductivity}

In this subsection, we intend to study the low frequency behavior of the optical conductivity, and give some insights into the coherent/incoherent behavior affected by the momentum dissipation and the Weyl term.

The optical conductivity studied in [17] shows a peak at low frequency for $\gamma>0$, but it actually shifts from the standard Drude formula if we require $\gamma \in \mathcal{S}_{0}$. Inspired by the incoherent metallic phase studied in [60] (also see [50, 61-65] for the related studies), we shall use the following modified Drude formula to fit the numerical data

$\sigma(\hat{\omega})=\frac{K \tau}{1-i \hat{\omega} \tau}+\sigma_{Q}$

where $K$ is a constant, $\tau$ the relaxation time and $\sigma_{Q}$ characters the incoherent degree. The left plot in Fig. 2 exhibits such incoherent non-Drude behavior with $\sigma_{Q}=0.866$ for $\gamma=1 / 12$. Note that for the cases with $\gamma$ far beyond the upper bound, i.e., $\gamma \gg 1$, we shall have a coherent Drude behavior (see the right plot in Fig. 2). Though there may be certain instabilities due to the violation of causality for $\gamma \gg 1$, the results presented in Fig. 2 can shed light on what modifications of the Drude formula of our model. As revealed in $[66,67]$, the weak momentum dissipation results in a Drude 

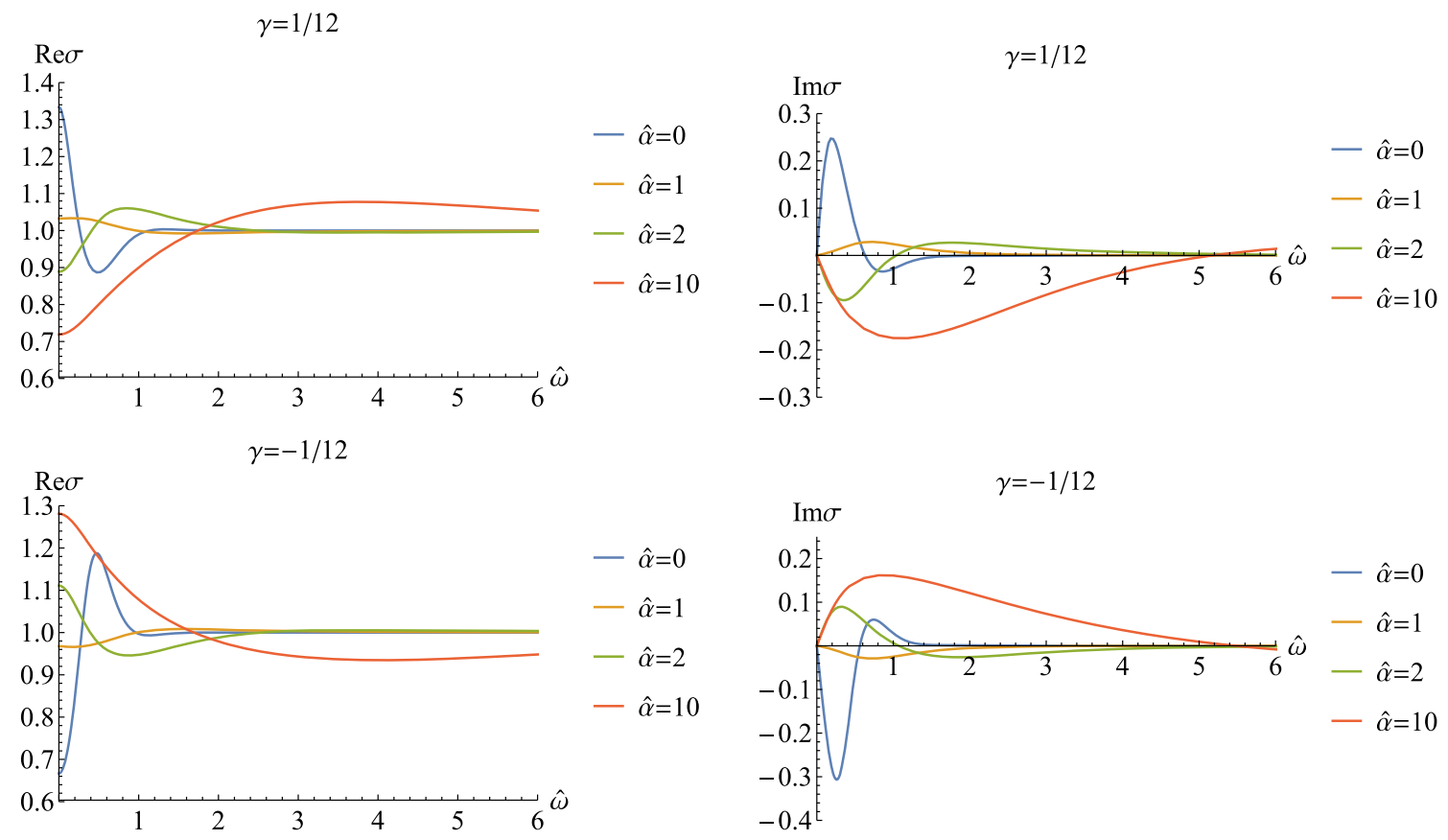

Fig. 1 The optical conductivity $\sigma(\hat{\omega})$ as the function of $\hat{\omega}$ with different $\hat{\alpha}$ for fixed $\beta$ (the plots above is for $\gamma=1 / 12$ and the one below for $\gamma=-1 / 12$ ). (For interpretation of the references to color in this figure, the reader is referred to the web version of this paper)

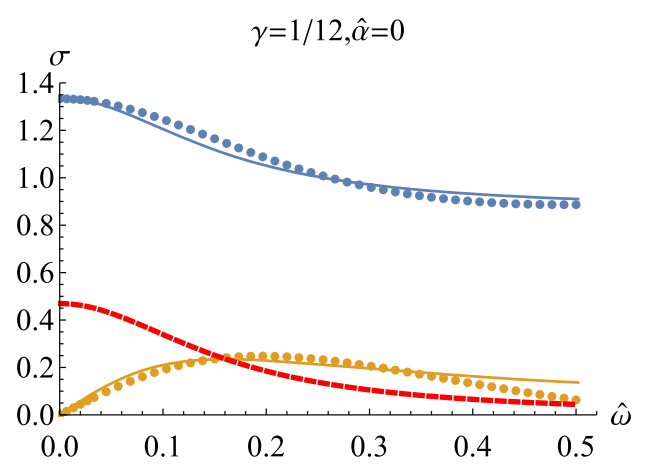

Fig. 2 The low frequency behavior of the optical conductivity without momentum dissipation for $\gamma=1 / 12$ (left plot) and $\gamma=10$ (right plot). The left plot is fitted by a modified Drude formula (22). The red dashed line in left plot is the real part of optical conductivity when fitted with the

peak, and with the increase of the momentum dissipation, the low frequency behavior of the conductivity deviates from the standard Drude formula and so it is depicted by the formula (22). But as we have already seen, that cannot be the case in the Maxwell-Weyl system (2). For large $\gamma$, the low frequency behavior of the conductivity can be described by the standard Drude formula (the right plot in Fig. 2). While for small $\gamma(\gamma>0)$, it cannot be depicted by the standard Drude formula but approximately by the modified Drude formula (22) (the left plot in Fig. 2). Therefore, to some degree, the Weyl term with $\gamma>0$ in our model may play the similar

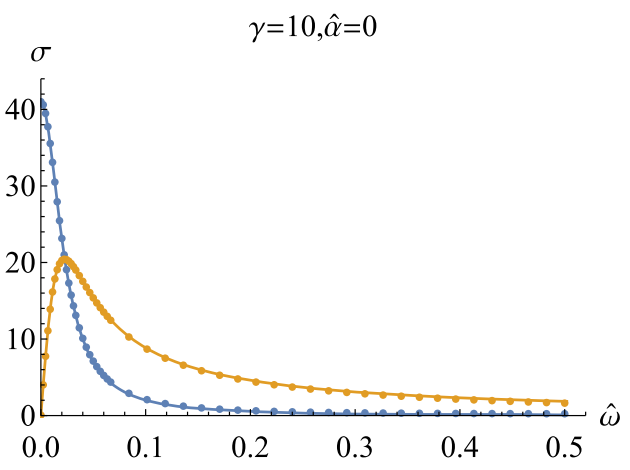

standard Drude formula. The right plot is fitted by the standard Drude formula. (For interpretation of the references to color in this figure, the reader is referred to the web version of this paper)

role as the momentum dissipation, but the equivalence need further explore. ${ }^{7}$

Furthermore, we fit the low frequency behavior of $\sigma(\hat{\omega})$ for different $\hat{\alpha}$ with fixed $\gamma=1 / 12$ (Fig. 3). We list the

\footnotetext{
${ }^{7}$ In [68], the authors have demonstrated that the low frequency conductivity in probe brane models exhibits Drude behavior. In particular, they proved that the low temperature hydrodynamics at linearized level is mathematically equivalent to the one of an ordinary fluid with weak momentum relaxation. Along this line, it is hopeful to address the low frequency behavior of our present model.
} 

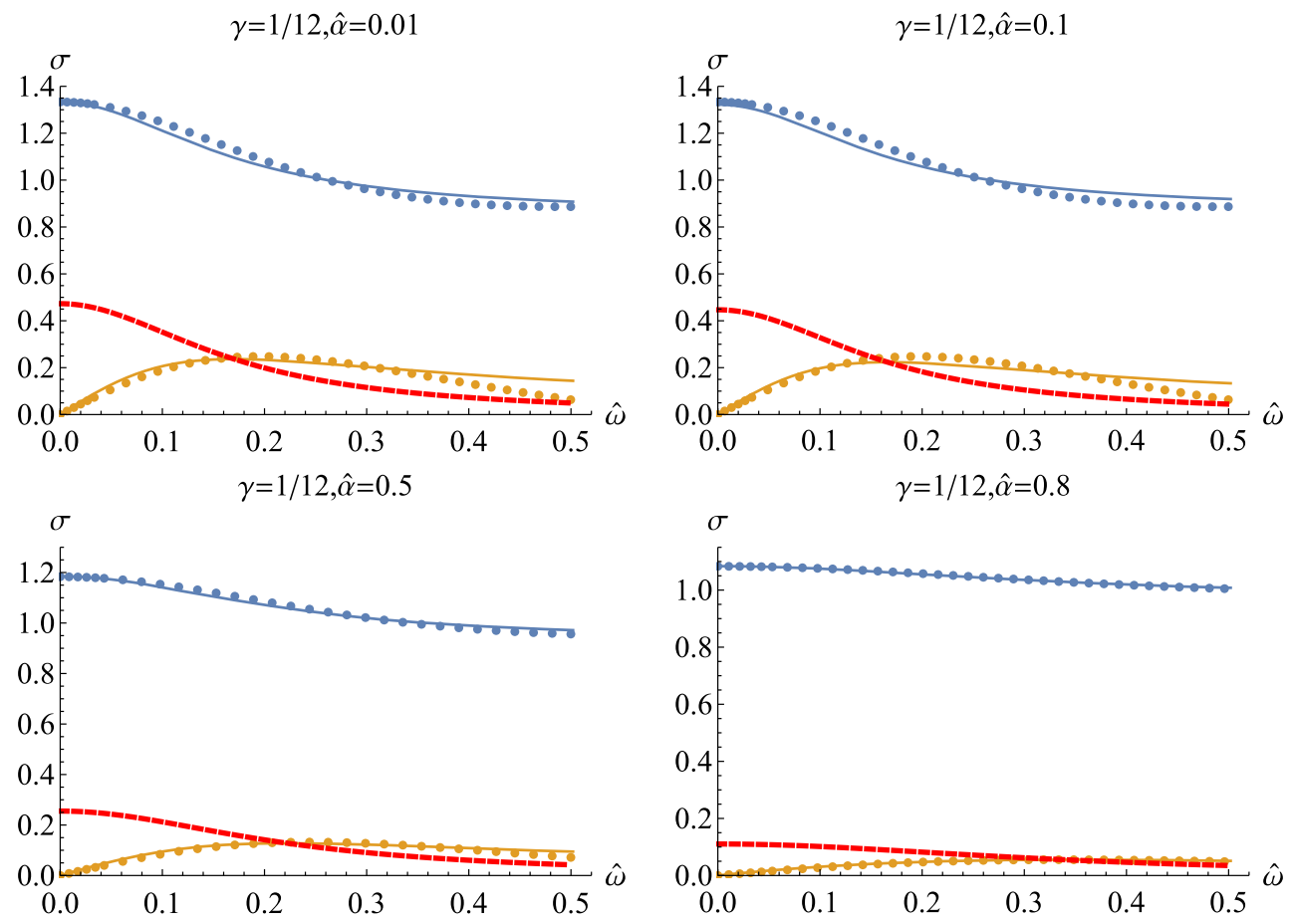

Fig. 3 The low frequency behavior of the optical conductivity with momentum dissipation for different $\hat{\alpha}$ and $\gamma=1 / 12$. They are fitted by a modified Drude formula (22). The red dashed line is the real part of

optical conductivity fitted with the standard Drude formula. (For interpretation of the references to color in this figure, the reader is referred to the web version of this paper)

Table 1 The characteristic quantity of incoherence $\sigma_{Q}$ for $\gamma=1 / 12$ and different $\hat{\alpha}$

\begin{tabular}{lllllll}
\hline$\hat{\alpha}$ & 0 & 0.01 & 0.05 & 0.1 & 0.5 & 0.8 \\
\hline$\sigma_{Q}$ & 0.866 & 0.859 & 0.865 & 0.875 & 0.929 & 0.973 \\
\hline
\end{tabular}

characteristic quantity of incoherence $\sigma_{Q}$ in Table 1 . Two illuminating features are summarized as follows.

- $\sigma_{Q}$ increases with the increase of $\hat{\alpha}$ in our present model (Table 1), which indicates that the incoherent behavior becomes more evident. Note that for small momentum dissipation $(\hat{\alpha}<0.06), \sigma_{Q}$ is smaller than that in SS-AdS case. This is reasonable because the small $\hat{\alpha}$ produces coherent contribution, which reduces the incoherent part from the Weyl term. With the increase of $\hat{\alpha}$, the stronger momentum dissipation combining with the contributions from Weyl term makes the system exhibit more prominent incoherent behavior.

- The fitting for large $\hat{\alpha}$ is better than that for small $\hat{\alpha}$ (Fig. 3) because for small $\hat{\alpha}$, the incoherent contribution mainly comes from the Weyl term. The phenomenon also implies that we may need a new non-Drude formula to meticulously depict the incoherent contribution from the Weyl

term with $\gamma>0$. We shall further explore this question in future.

We turn to check the low frequency behavior for $\gamma<0$ and large $\hat{\alpha}$, in which cases a peak exhibits (see the bottom plots in Fig. 1). From Fig. 4, we see that the non-Drude behavior can be well fitted with different $\hat{\alpha}$ for $\gamma=-1 / 12$ by (22). The fitted $\sigma_{Q}$ with different $\hat{\alpha}$ for $\gamma=-1 / 12$ are listed in Table 2.

In summary, both the Weyl coupling and the momentum dissipation contributes to the modifications of the Drude formula. And their definitive roles deserve further exertion.

\section{DC conductivity, diffusion constant and susceptibility}

In this section, we study the DC conductivity, charge diffusion constant and susceptibility accommodating with the Maxwell-Weyl theory (2) with the momentum dissipation.

There are many ways to calculate these quantities. Here we shall use the membrane paradigm approach $[69,70]$. The key point of the membrane paradigm is to define the membrane current on the stretched horizon $u_{H}=1-\epsilon$ with $\epsilon \ll 1$

$j^{\mu}=\left.\frac{1}{4} n_{v} X^{\mu \nu \rho \sigma} F_{\rho \sigma}\right|_{u=u_{H}}$, 

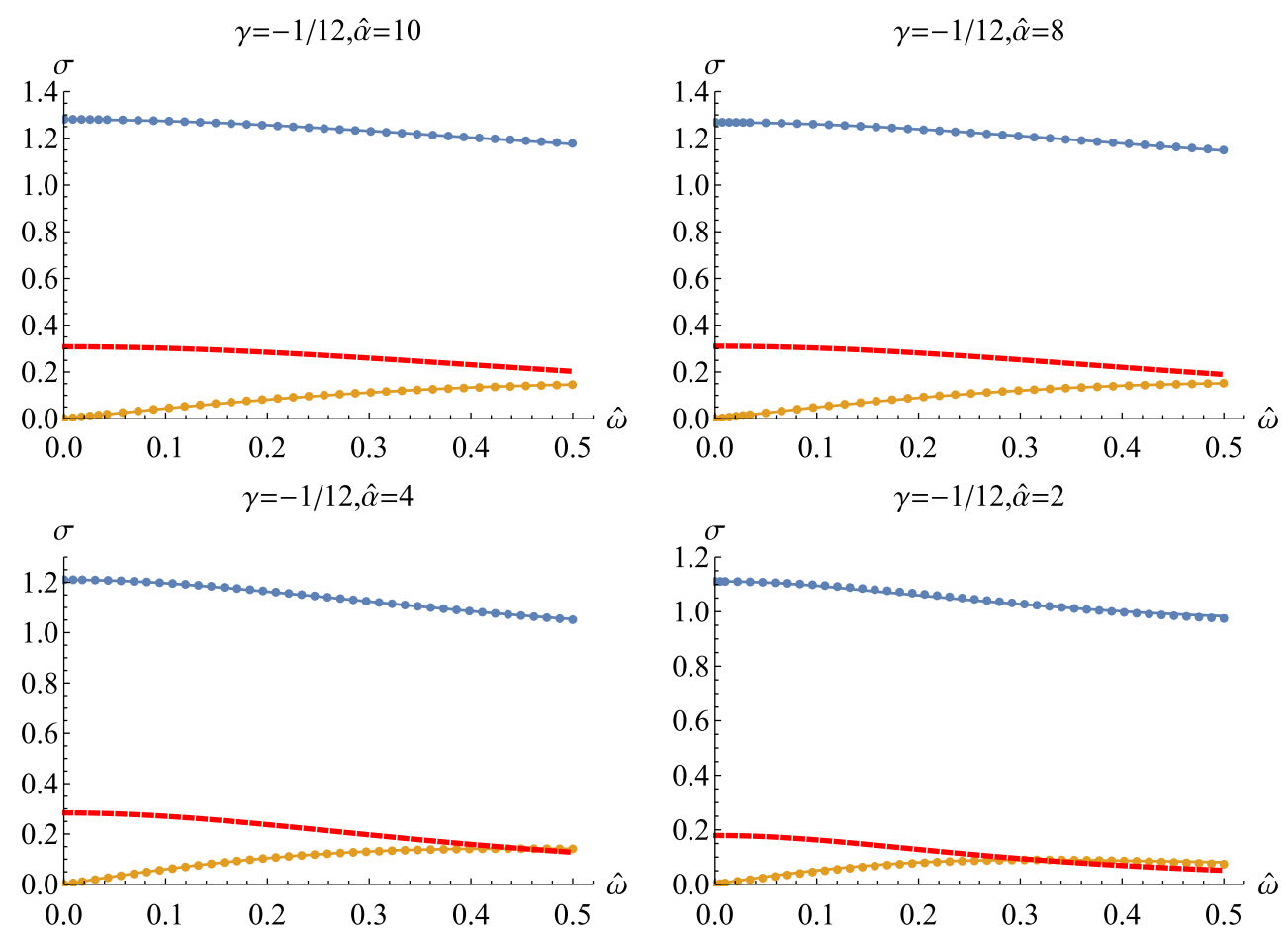

Fig. 4 The low frequency behavior of the optical conductivity with momentum dissipation for different $\hat{\alpha}$ and $\gamma=-1 / 12$. They are fitted by a modified Drude formula (22). The red dashed line is the real part of

optical conductivity fitted with the standard Drude formula. (For interpretation of the references to color in this figure, the reader is referred to the web version of this paper)

Table 2 The characteristic quantity of incoherence $\sigma_{Q}$ for $\gamma=-1 / 12$ and different $\hat{\alpha}$

\begin{tabular}{llllll}
\hline$\hat{\alpha}$ & 10 & 8 & 6 & 4 & 2 \\
\hline$\sigma_{Q}$ & 0.972 & 0.957 & 0.941 & 0.926 & 0.932
\end{tabular}

where $n_{v}$ is a unit radial normal vector. By the Ohm's law, it is straightforward to write down the expression of the DC conductivity in our present framework $[17,47]$

$\sigma_{0}=\left.\sqrt{-g} g^{x x} \sqrt{-g^{t t} g^{u u} X_{1} X_{5}}\right|_{u=1}$.

Moreover, following [69,70], it is also easy to obtain the diffusion constant $[17,47]$

$D=-\sigma_{0} \int_{0}^{1} \frac{1}{\sqrt{-g} g^{t t} g^{u u} X_{3}} d u$.

Then, using the Einstein relation $D=\sigma_{0} / \chi$, the susceptibility can be expressed as $[69,70]$

$\chi^{-1}=-\int_{0}^{1} \frac{1}{\sqrt{-g} g^{t t} g^{u u} X_{3}} d u$.

For the details, one can refer to [17,47]. For the diffusion constant and the susceptibility, we mainly focus on the dimensionless quantities $\hat{D} \equiv 2 \pi T D$ and $\hat{\chi} \equiv 2 \pi T \chi$. In the following, we shall explicitly discuss these quantities.

$\sigma_{0}=1+\frac{2}{3}\left(2+\frac{4\left(\sqrt{6 \hat{\alpha}^{2}+1}-2 \hat{\alpha}^{2}-1\right)}{\hat{\alpha}^{2}}\right) \gamma$

It is obvious that the DC conductivity is linear dependence on $\gamma$ for given $\hat{\alpha}$ (Eq. (27) or see left plot in Fig. 5). Also we note that when $\hat{\alpha}=2 / \sqrt{3}, \sigma_{0}=1$ is independent of the Weyl coupling parameter $\gamma$. It is a specific point of the conductivity and we will discuss later. More explicitly, in Fig. 5, we show how $\sigma_{0}$ behaves as $\hat{\alpha}$ with fixed $\gamma$ in the left plot and as $\gamma$ with fixed $\hat{\alpha}$ in right plot. Since the momentum dissipation destroys the motion of the particle (vortices), $\sigma_{0}$ decreases (increases) as $\hat{\alpha}$ increases for $\gamma>0(\gamma<0)$. This picture is consistent with that for the optical conductivity discussed in previous section. Moreover, we would like to emphasize that, although the DC conductivity $\sigma_{0}=1$ with $\hat{\alpha}=2 / \sqrt{3}$ independent of $\gamma$, the optical conductivity still depends on the Weyl parameter beyond the points $\omega \rightarrow 0$ and $\infty$ (see Fig. 6). 


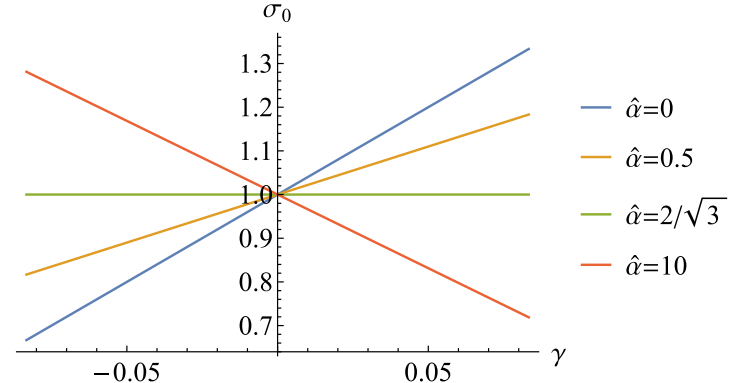

Fig. 5 Left plot: the DC conductivity $\sigma_{0}$ versus the Weyl coupling parameter $\gamma$ for some fixed dissipation constant $\hat{\alpha}$. Right plot: the DC conductivity $\sigma_{0}$ versus the dissipation constant $\hat{\alpha}$ for some fixed Weyl

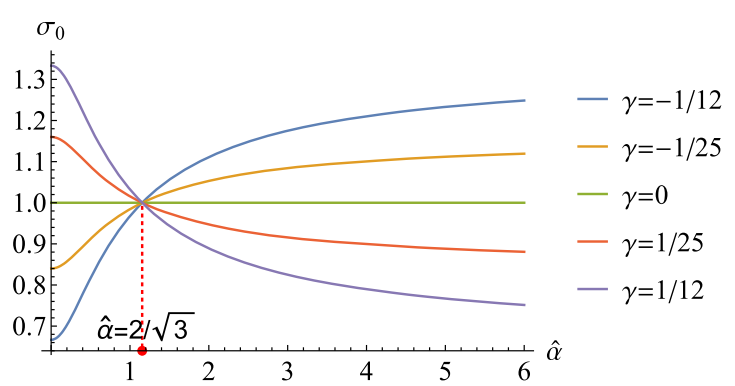

coupling parameter $\gamma$. (For interpretation of the references to color in this figure, the reader is referred to the web version of this paper)
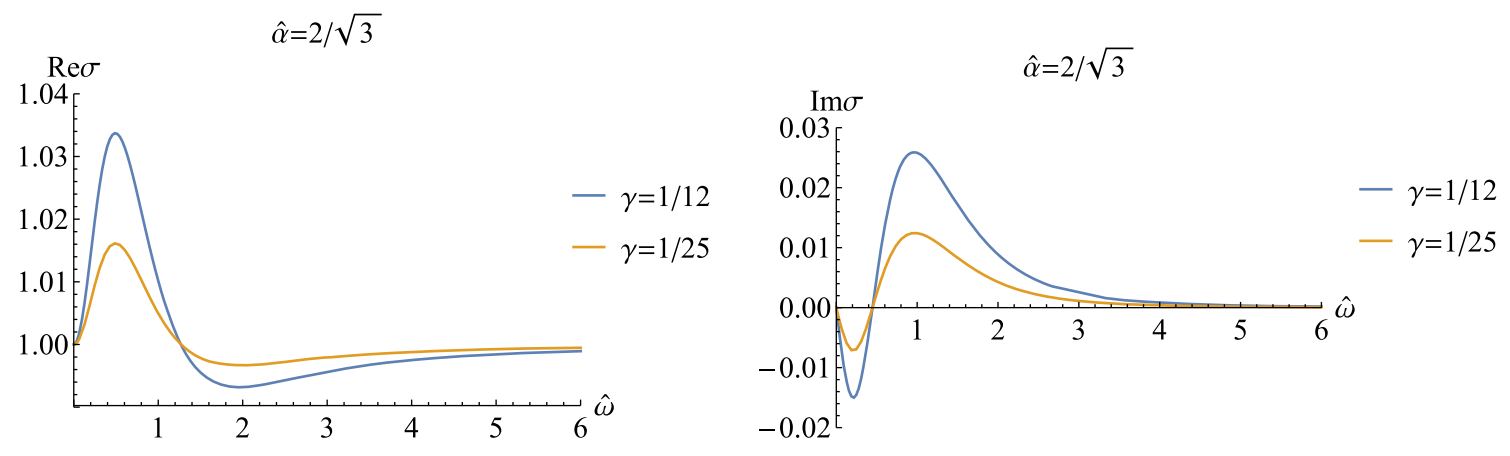

Fig. 6 The real part (left plot) and the imaginary part (right plot) of the optical conductivity as the function of $\hat{\omega}$ for $\hat{\alpha}=2 / \sqrt{3}$ and different values of $\gamma$. (For interpretation of the references to color in this figure, the reader is referred to the web version of this paper)

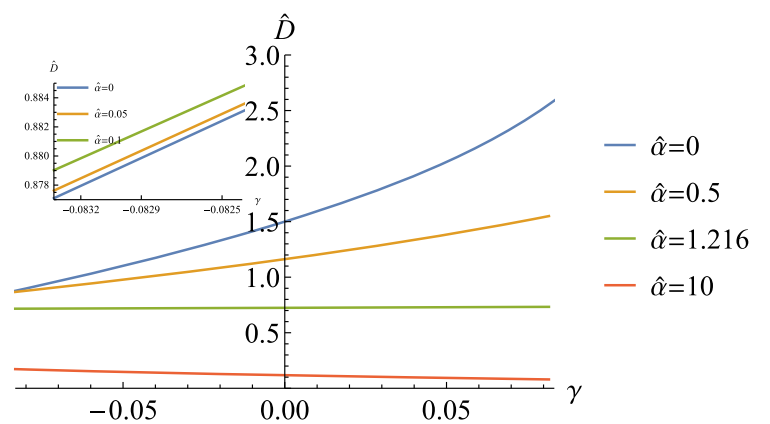

Fig. 7 Left plot: the charge diffusion constant $\hat{D}$ versus the Weyl coupling parameter $\gamma$ for some fixed dissipation constant $\hat{\alpha}$. Right plot: the charge diffusion constant $\hat{D}$ versus the dissipation constant $\hat{\alpha}$ for some

\subsection{Diffusion constant and susceptibility}

The diffusion constant $\hat{D}$ and the susceptibility $\hat{\chi}$ with Weyl correction in the boundary field theory dual to the SS-AdS geometry can be analytically worked out $[17,47]$. But in our case it is difficult to analytically derive their expressions. Instead, we shall first present the numerical results. And then we would like to find perturbative corrections to $\hat{D}$ and $\hat{\chi}$ in $\gamma$ and $\hat{\alpha}$, which could confirm and clarity some features of the numerical results.

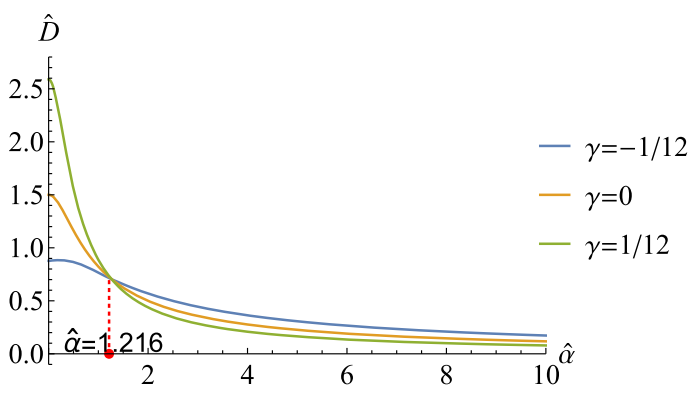

fixed Weyl coupling parameter $\gamma$. (For interpretation of the references to color in this figure, the reader is referred to the web version of this paper)

\subsubsection{Numerical results}

The numerical results for $\hat{D}$ and $\hat{\chi}^{-1}$ are shown in Figs. 7 and 8 , respectively. In the left plots, we study their dependence on $\gamma$ by fixing some $\hat{\alpha}$ and we focus on their dependence on $\hat{\alpha}$ with fixed samples of $\gamma$ in the right plots. According to the figures, we summarize the properties of $\hat{D}$ and $\hat{\chi}^{-1}$ as follows. 


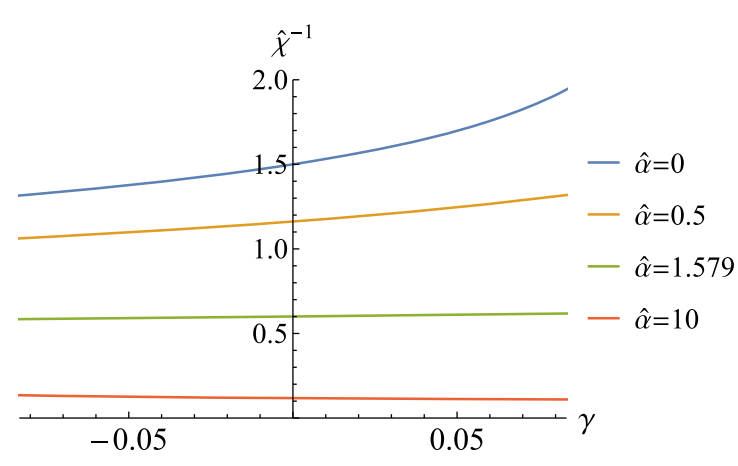

Fig. 8 Left plot: the inverse of the susceptibility $\hat{\chi}^{-1}$ versus the Weyl coupling parameter $\gamma$ for some fixed dissipation constant $\hat{\alpha}$. Right plot: the inverse of the susceptibility $\hat{\chi}^{-1}$ versus the dissipation constant $\hat{\alpha}$

1. Similar to the feature of the DC conductivity, there also exists specific values of $\hat{\alpha}$, with which $\hat{D}$ and $\hat{\chi}^{-1}$ are independent of the Weyl coupling parameter. The specific values are $\hat{\alpha} \approx 1.216$ for $\hat{D}$ and $\hat{\alpha} \approx 1.578$ for $\hat{\chi}^{-1}$, respectively. We note that for different observables, $\sigma_{0}$, $\hat{D}$ and $\hat{\chi}^{-1}$, the specific values of $\hat{\alpha}$ are different and so these specific values are not universal.

2. When $\hat{\alpha}<1.216$, the diffusion constant increases with the increase of $\gamma$. But the tendency of the increase of the $\hat{D}$ as the function $\gamma$ tends to slow down with the increase of $\hat{\alpha}$. While for $\hat{\alpha}>1.216$, the case is opposite, i.e., with the increase of $\gamma, \hat{D}$ decreases, although the tendency of the decrease is weak. Similar phenomena can be found for the susceptibility.

3. $\hat{\chi}^{-1}$ is suppressed by the momentum dissipation, so does the diffusion constant $\hat{D}$. It implies that for the particles excitation and vortices excitation, the momentum dissipation has similar effect on $\hat{D}$ and $\hat{\chi}^{-1}$. But there is exception that when $\gamma$ approaches the low bound and $\hat{\alpha}$ is small, $\hat{D}$ becomes large with the increase of $\hat{\alpha}$ (see the inset in left plot of Fig. 7 and the right plot of Fig. 7). We shall further understand this phenomenon and their microscopic mechanism in future.

\subsubsection{Analytic results}

Let's continue to present available analytic. Even though it is difficult to derive the exact analytical results of $\hat{D}$ and $\hat{\chi}$, we can derive the perturbative corrections to them in $\gamma$ and $\hat{\alpha}$. We first study the perturbative corrections to $\hat{D}$ and $\hat{\chi}$ in $\gamma$. Up to the first order, they are given, respectively, by

$$
\begin{aligned}
\hat{D}= & \frac{\sqrt{6 \hat{\alpha}^{2}+1}-1}{2 \hat{\alpha}^{2}} \\
& -\frac{2\left(12 \sqrt{6 \hat{\alpha}^{2}+1} \hat{\alpha}^{2}-63 \hat{\alpha}^{2}+17 \sqrt{6 \hat{\alpha}^{2}+1}-17\right) \gamma}{9 \hat{\alpha}^{4}},
\end{aligned}
$$

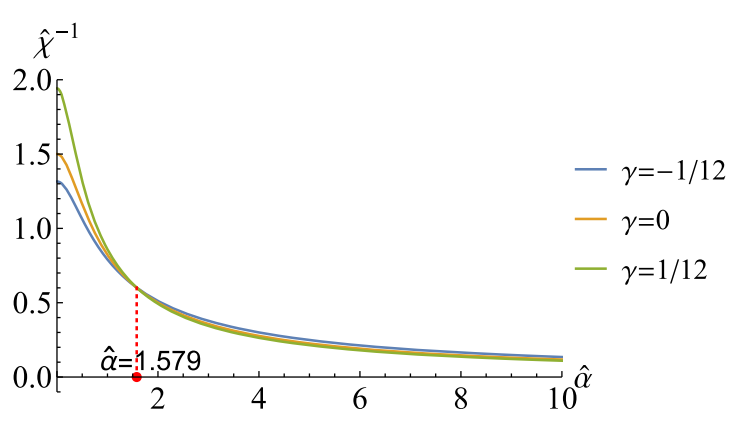

for some fixed Weyl coupling parameter $\gamma$. (For interpretation of the references to color in this figure, the reader is referred to the web version of this paper)

$$
\begin{aligned}
\hat{\chi}^{-1}= & \frac{\sqrt{6 \hat{\alpha}^{2}+1}-1}{2 \hat{\alpha}^{2}} \\
& +\frac{\left(10\left(\sqrt{6 \hat{\alpha}^{2}+1}-1\right)-12 \hat{\alpha}^{2}\right)\left(\sqrt{6 \hat{\alpha}^{2}+1}-1\right) \gamma}{18 \hat{\alpha}^{4}} .
\end{aligned}
$$

Immediately, from the above equations, we can find that when $\hat{\alpha}=\frac{\sqrt{51}}{4 \sqrt{2}} \approx 1.26\left(\hat{\alpha}=\sqrt{\frac{5}{2}} \approx 1.58\right)$, Both $\hat{D}$ and $\hat{\chi}^{-1}$ are independent of the Weyl coupling parameter $\gamma$. The analytical results are in good agreement with the numerical results shown in previous subsection.

To further see the effect of $\gamma$ on $\hat{D}$ and $\hat{\chi}^{-1}$, we extract the coefficients of $\gamma$ in Eqs. (30) and (31). The coefficients are denoted as $\frac{d \hat{D}}{d \gamma}$ and $\frac{d \hat{\chi}^{-1}}{d \gamma}$ and they only depend on the momentum relaxation. Their explicit behavior are shown in Fig. 9, from which we see that as $\hat{\alpha}$ increases, both $\frac{d \hat{D}}{d \gamma}$ and $\frac{d \hat{\chi}^{-1}}{d \gamma}$ first decrease sharply and then increase smoothly. This tendency are consistent with the observations in Figs. 7 and 8 and the second features we described in last subsection.

Then we derive the perturbative corrections to $\hat{D}$ and $\hat{\chi}^{-1}$ in $\hat{\alpha}$, which are

$$
\begin{aligned}
\hat{D} & =\hat{D}_{0}+\hat{D}_{2} \hat{\alpha}^{2}, \\
\hat{\chi}^{-1} & =\hat{\chi}_{0}^{-1}+\hat{\chi}_{2}^{-1} \hat{\alpha}^{2},
\end{aligned}
$$

with

$$
\begin{aligned}
& \hat{D}_{0}= \frac{(4 \gamma+1)\left(\log \left(\frac{1-8 \gamma}{(1-2 \sqrt[3]{\gamma})^{3}}\right)-2 \sqrt{3} \tan ^{-1}\left(\frac{\sqrt[3]{\gamma}+1}{\sqrt{3} \sqrt[3]{\gamma}}\right)+\sqrt{3} \pi\right)}{8 \sqrt[3]{\gamma}} \\
& \hat{D}_{2}=\left(\gamma ^ { 2 / 3 } \left(\frac{3}{2} \log \left(\frac{(1-2 \sqrt[3]{\gamma})^{3}}{1-8 \gamma}\right)\right.\right.
\end{aligned}
$$



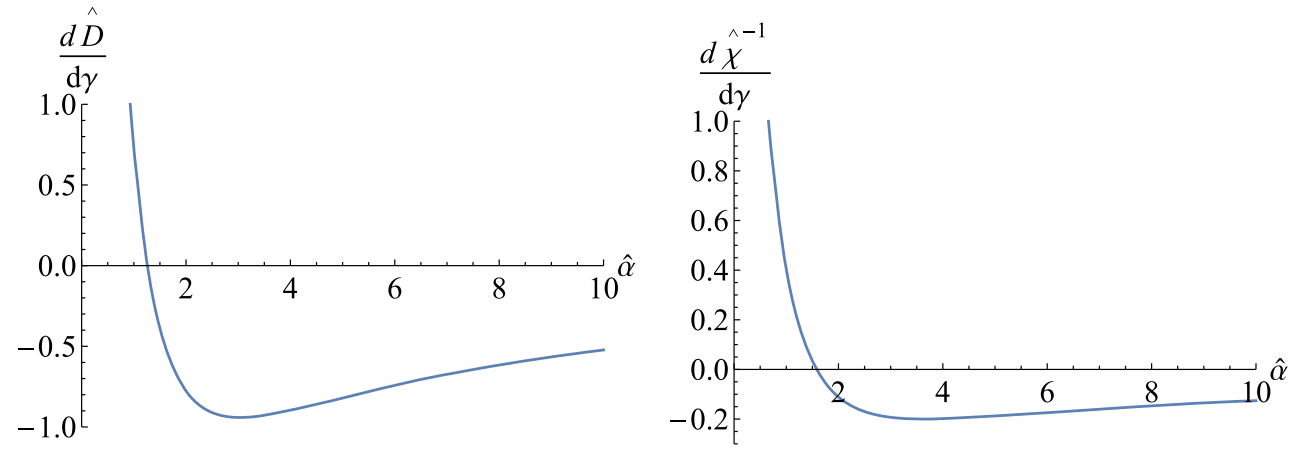

Fig. 9 Left plot: $\frac{d \hat{D}}{d \gamma}$ as the function of $\hat{\alpha}$. Right plot: $\frac{d \hat{\chi}^{-1}}{d \gamma}$ as the function of $\hat{\alpha}$

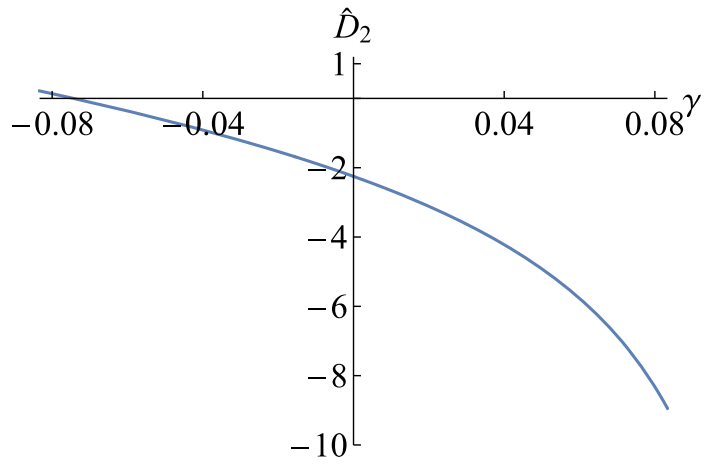

Fig. $10 \hat{D}$ as the function of $\gamma$ in the allowed region of $\gamma$

$$
\begin{aligned}
& \left.-3 \sqrt{3} \tan ^{-1}\left(\frac{4 \sqrt[3]{\gamma}+1}{\sqrt{3}}\right)+\frac{\sqrt{3} \pi}{2}\right) \\
& \left.-3 \gamma+\frac{3(4 \gamma+1)}{2(8 \gamma-1)}-\frac{3}{4}\right), \\
\hat{\chi}_{0}^{-1}= & \frac{\log \left(\frac{1-8 \gamma}{(1-2 \sqrt[3]{\gamma})^{3}}\right)-2 \sqrt{3} \tan ^{-1}\left(\frac{\sqrt[3]{\gamma}+1}{\sqrt{3} \sqrt[3]{\gamma}}\right)+\sqrt{3} \pi}{12 \sqrt[3]{\gamma}}, \\
\hat{\chi}_{2}^{-1}= & \frac{9-24 \gamma}{32 \gamma-4} .
\end{aligned}
$$

The above results of $\hat{D}_{0}$ and $\hat{\chi}_{0}^{-1}$ are consistent with the ones without axions [17]. In addition, it is straightforward from Eq. (35) that $\hat{\chi}<0$ in the allowed region of $\gamma$, which means that the momentum dissipation suppresses $\hat{\chi}^{-1}$, just as found in the above subsection.

Furthermore, to explicitly see the affect of momentum relaxation on diffusion, we plot $\hat{D}_{2}$ as the function of $\gamma$ in Fig. 10. For most allowed region of $\gamma, \hat{D}_{2}$ is negative, which indicates that the momentum dissipation suppress the diffusion constant. When $\gamma$ approaches the low bound, $\hat{D}_{2}$ becomes positive, which means the effect of the momentum dissipation is opposite. The analytic analysis gives the same conclusion as the numerical one.

It would be very interesting in resorting to another analytical approach to our present theory by computing quasinormal modes as in [71]. It may provide more insight into the features that we observed here. We leave this for future.

\section{EM duality}

It has been illustrated in Sect. 2 (see also Appendix A or [17]) that for very small $\gamma$, the original EM theory relates to its dual theory by changing the sign of $\gamma$. In the AdS/CFT correspondence, the bulk EM duality corresponds to the particle-vortex duality in the boundary field theory, in which the optical conductivity in the dual theory is the inverse of that in its original theory ${ }^{8}[17,18]$

$\sigma_{*}(\hat{\omega} ; \hat{\alpha}, \gamma)=\frac{1}{\sigma(\hat{\omega} ; \hat{\alpha}, \gamma)}$

Since for small $\gamma$, the change of the sign of $\gamma$ corresponds to an approximate particle-vortex duality effect, so we have [17-22]

$\sigma(\hat{\omega} ; \hat{\alpha}, \gamma) \approx \frac{1}{\sigma(\hat{\omega} ; \hat{\alpha},-\gamma)}, \quad|\gamma| \ll 1$.

From Eqs. (36) and (37), we can conclude the following relation

$\sigma_{*}(\hat{\omega} ; \hat{\alpha}, \gamma) \approx \sigma(\hat{\omega} ; \hat{\alpha},-\gamma), \quad|\gamma| \ll 1$

The above equation indicates that the optical conductivity in the dual EM theory is approximately equal to that in its original theory for the opposite sign of $\gamma$. It also has been explicitly illustrated for $\gamma= \pm 1 / 12$ in Fig. 5 in [17], from which we can obviously see that the conductivity of the dual EM theory is not precisely equal to that of its original theory for the oppositive sign of $\gamma$ except for $\hat{\omega} \rightarrow \infty$. Next, we shall explore the effect of the momentum dissipation on the EM duality by explicitly presenting the frequency dependent conductivity of the original theory and its dual theory.

\footnotetext{
8 The proof of this relation can be found in $[17,18]$, and on can also refer to [24] for the derivation of the relation in a specific class of CFTs.
} 

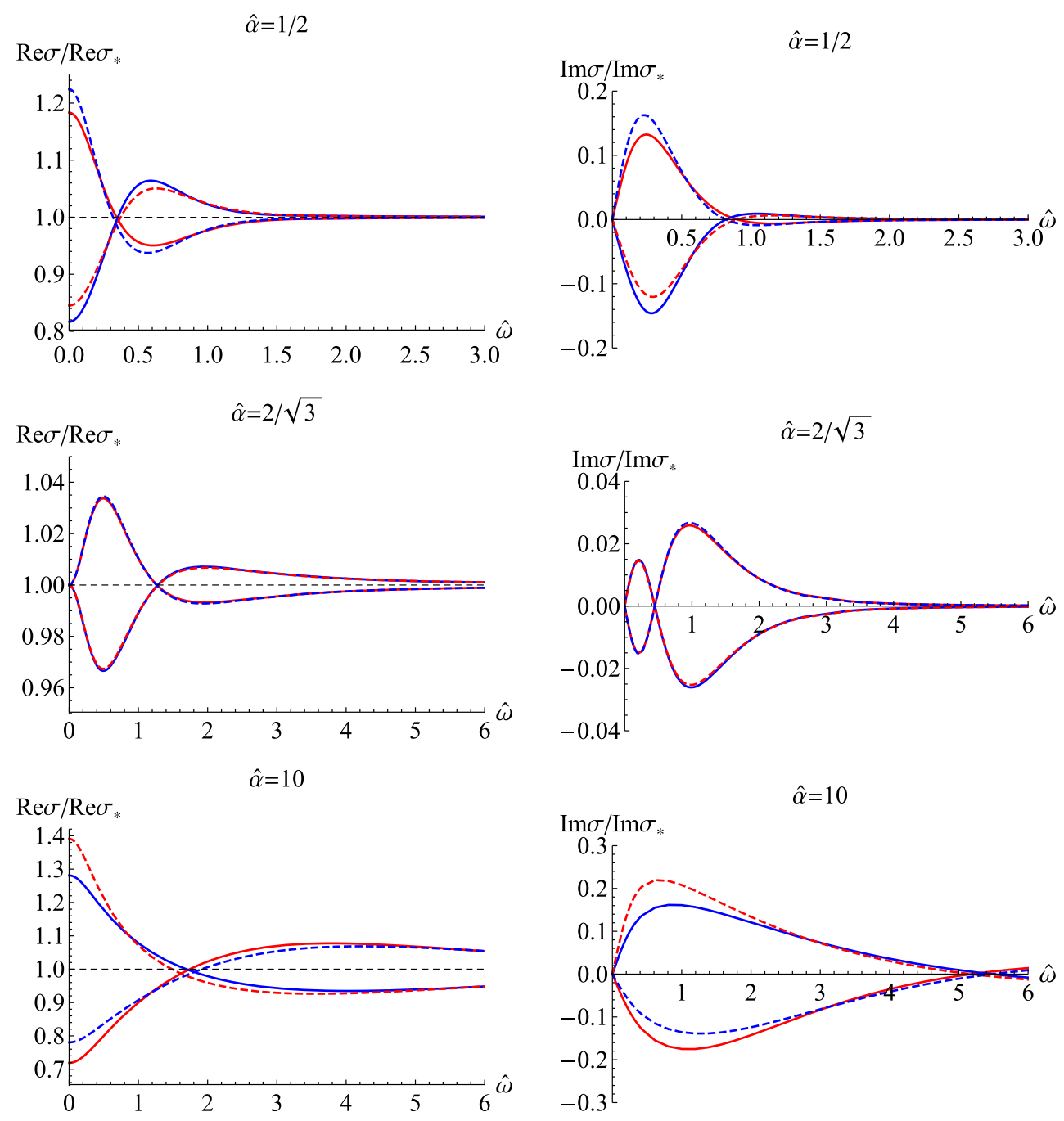

Fig. 11 The real part (left plot) and the imaginary part (right plot) of the optical conductivity as the function of $\hat{\omega}$ for various values of $\gamma$ and $\hat{\alpha}$. The solid curves are the conductivity of the original EM theory (3), which have been shown previously in Fig. 1 (red for $\gamma=1 / 12$ and blue

We firstly focus on the DC conductivity, which can be analytically derived [see Eq. (27)]. To this end, we also derive the DC conductivity of the dual EM theory (7) as

$$
\begin{aligned}
\sigma_{* 0}(\hat{\alpha}, \gamma) & =\frac{1}{1+\frac{2}{3}\left(2+\frac{4\left(\sqrt{6 \hat{\alpha}^{2}+1}-2 \hat{\alpha}^{2}-1\right)}{\hat{\alpha}^{2}}\right) \gamma} \\
& \approx 1-\frac{2}{3}\left(2+\frac{4\left(\sqrt{6 \hat{\alpha}^{2}+1}-2 \hat{\alpha}^{2}-1\right)}{\hat{\alpha}^{2}}\right) \gamma
\end{aligned}
$$

The second line $(\approx)$ is for $|\gamma| \ll 1$. Similarly with $\sigma_{0}$, we have a specific value $\hat{\alpha}=2 / \sqrt{3}$, for which $\sigma_{* 0}=1$ and is independent of $\gamma$. Specifically, at this value of $\hat{\alpha}$, we have $\sigma_{* 0}=\sigma_{0}=1$.

for $\gamma=-1 / 12$ ). While the dashed curves display the conductivity of the EM dual theory (7) for the same value of $\gamma$ and $\hat{\alpha}$. (For interpretation of the references to color in this figure, the reader is referred to the web version of this paper)

Subsequently, we turn to study the optical conductivity. Fig. 11 displays the real and imaginary part of the optical conductivity of the bulk EM theory and its dual EM theory as the function of the frequency $\hat{\omega}$ for $\gamma= \pm 1 / 12$ and various values of $\hat{\alpha}$. As expected, an oppositive picture appears in the dual EM theory. That is to say, for small $\hat{\alpha}$, a peak occurs at small frequency for $\gamma=-1 / 12$ while a dip exhibits for $\gamma=1 / 12$. While for large $\hat{\alpha}$, the phenomenon is opposite. In addition, Fig. 11 also further illustrates that the relation (37) or (38) holds only for $|\gamma| \ll 1$.

Also we find that at the specific value of $\hat{\alpha}=2 / \sqrt{3}$, the optical conductivity of the original theory is almost (approximate but not exact) the same as that of the dual theory when the sign of $\gamma$ changes. It indicates that the particle-vortex duality holds very well at $\hat{\alpha}=2 / \sqrt{3}$. We would like to 


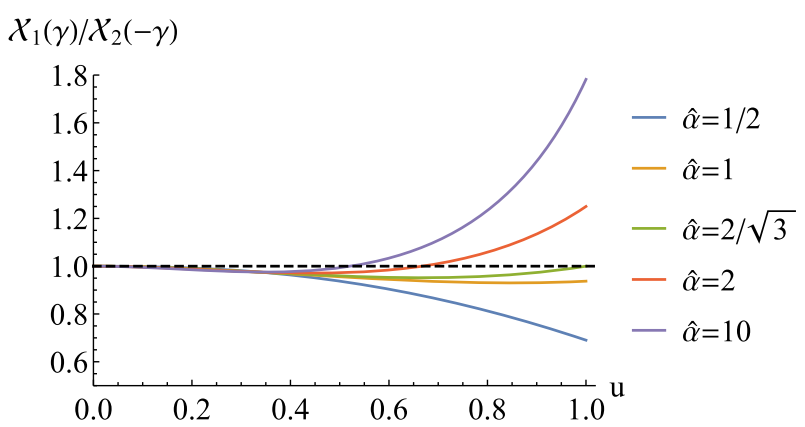

Fig. $12 \mathcal{X}_{1}(\gamma) / \mathcal{X}_{2}(-\gamma)$ as the function of $u$ with different $\hat{\alpha}$. Here we set $\gamma=1 / 12$

point out that the specific point $\hat{\alpha}=2 / \sqrt{3}$ is not the selfduality point of our model because at this point, we don't have $\hat{X}=X$ such that the dual theory (7) is not identical with its original theory (3). It also indicates that there isn't some emergent symmetry in the spectrum at this specific value. Therefore, this point is different from the point found in the thermal conductivity of the Maxwell theory in EA-AdS geometry, at which the thermal conductivity is independent of the frequency [52]. At present, it is hard to obtain an analytical understanding on this issue at the special value of $\hat{\alpha}=2 / \sqrt{3}$, but we attempt to taste kind of analytical similarity between the equation of motion (A6) and its dual one with zero momentum, which are

$$
\begin{aligned}
& A_{y}^{\prime \prime}+\left(\frac{f^{\prime}}{f}+\frac{X_{6}^{\prime}}{X_{6}}\right) A_{y}^{\prime}+\frac{\mathfrak{p}^{2}}{f^{2}} \frac{X_{2}}{X_{6}} \hat{\omega}^{2} A_{y}=0, \\
& A_{y}^{\prime \prime}+\left(\frac{f^{\prime}}{f}-\frac{X_{6}^{\prime}}{X_{6}}\right) A_{y}^{\prime}+\frac{\mathfrak{p}^{2}}{f^{2}} \frac{X_{6}}{X_{2}} \hat{\omega}^{2} A_{y}=0 .
\end{aligned}
$$

Since $\frac{X_{2}}{X_{6}}=\frac{X_{6}}{X_{2}}$ for any $\hat{\alpha}$, so the difference between the above equations exists in the coefficients of $A_{y}^{\prime}$, which are

$$
\begin{aligned}
& \mathcal{X}_{1}(\gamma) \equiv \frac{X_{6}^{\prime}}{X_{6}}=\frac{-\frac{2}{3} \gamma u^{2} f^{(3)}(u)-\frac{4}{3} \gamma u f^{\prime \prime}(u)}{1-\frac{2}{3} \gamma u^{2} f^{\prime \prime}(u)}, \\
& \mathcal{X}_{2}(\gamma) \equiv-\frac{X_{6}^{\prime}}{X_{6}}=\frac{\frac{2}{3} \gamma u^{2} f^{(3)}(u)+\frac{4}{3} \gamma u f^{\prime \prime}(u)}{1-\frac{2}{3} \gamma u^{2} f^{\prime \prime}(u)} .
\end{aligned}
$$

To see the speciality of $\hat{\alpha}=2 / \sqrt{3}$, we show explicitly the behavior of $\frac{\mathcal{X}_{1}(1 / 12)}{\mathcal{X}_{2}(-1 / 12)}$ with different $\hat{\alpha}$ in Fig. 12. It is obvious that for $\hat{\alpha}=2 / \sqrt{3}$, the value of $\frac{\mathcal{X}_{1}(1 / 12)}{\mathcal{X}_{2}(-1 / 12)}$ approaches to 1 near the horizon, which implies a most similar between (40) and (41) among all $\hat{\alpha}$. On the other hand, it is well known that the low frequency behavior of the conductivity is mainly controlled by aspects near the horizon. Therefore, to some extent, the above comparison in the original equations of motion helps us understand the similarity of the optical conductivities at $\hat{\alpha}=2 / \sqrt{3}$ in the two theories with sign of $\gamma$ changed.

\section{Discussions and open questions}

In this paper, we have studied the transports, in particular the electric conductivity, in a neutral plasma with momentum dissipation dual to the Maxwell-Weyl system in EA-AdS geometry. In previous studies for the neutral system at finite temperature dual to the Maxwell-Weyl system in SS-AdS geometry [17-22], the authors found that the optical conductivity exhibits a peak or a dip depending on the sign of the Weyl coupling parameter $\gamma$. It provided a possible description of the CFT of the boson Hubbard model in holographic framework [25]. But there is still a degree of freedom of the sign of $\gamma$.

Our main results for the optical conductivity with the momentum dissipation were displayed in Fig. 1 and the corresponding physical interpretation was presented in Sect. 4 . For $\gamma>0$, the strong disorder drives the peak in the low frequency optical conductivity into a dip. While for $\gamma<0$, an opposite scenario is observed, that is to say, the dip in optical conductivity at low frequency gradually upgrades and eventually develops into a peak with the increase of the disorder. Our present model provides a route toward the problem that which sign of $\gamma$ is physically correct description of the CFT of boson Hubbard model. Also we have quantitatively studied the low frequency behavior of the optical conductivity by fitting our numerical data with the modified Drude formula (22). It provides a hint regarding the coherent or incoherent contribution from the Weyl term as well as the momentum dissipation, and deserves further studying.

Further, we found that there is a specific value of the momentum dissipation constant $\hat{\alpha}=2 / \sqrt{3}$, for which the DC conductivity $\sigma_{0}$ is independent of $\gamma$ and the particlevortex duality related by the change of the sign of $\gamma$ holds very well. Aside from the conductivity, there is also a specific value of $\hat{\alpha}$ for the diffusion constant and susceptibility, for which these quantities are independent of the Weyl coupling parameter $\gamma$. But these specific values of $\hat{\alpha}$ are different from each other and so they are not universal in this Maxwell-Weyl system in EA-AdS geometry.

In addition, we also present several comments on the physics of our present model as follows. References [17-22] introduce an extra Weyl tensor $C_{\mu \nu \rho \sigma}$ coupling to Maxwell field in the SS-AdS black brane to study the QC transports in the neutral plasma at finite temperature. Another way to study the QC physics in holographic framework is to couple the gravity with a neutral bulk scalar field, whose dual scalar operator has an expected value [72]. Then we study the QC physics by setting the source of scalar field in the dual boundary theory to zero [72]. If the source of scalar field does not vanish, the model of [72] also provides a starting point to study the physics away from QCP. In our present model, $\Phi_{I}$ correspond to turning on spatial linear sources which intro- 
duces a dimensional parameter $\alpha$ in the dual boundary theory, and so the physics we studied is away from QCP.

Finally, we comment some open questions deserving further exploration.

1. In this paper, we mainly study the optical conductivity at the zero momentum, which is relatively easy to calculate since the equations of motion are simplified into a great extent. But the transports at the finite momentum and energy in condensed matter laboratories have been obtained now or shall be given in near future [73-77], which reveal more information of the systems. On the other hand, in [19] they have also studied the responses of Maxwell-Weyl system in SS-AdS geometry at the finite momentum and found that it indeed provide far deeper insights into this system than that at the zero momentum. Therefore it is interesting and important to further study the responses of our present model with the momentum dissipation at full momentum and energy spaces.

2. The spatial linear dependent axionic fields are the simplest way to implement the momentum dissipation, or say disorder. We can also introduce the momentum dissipation by incorporating the higher order terms of axions [78-82] to study the properties of transport of the Maxwell-Weyl system. The higher order terms of axions induce metal-insulator transition (MIT) [81,82] and provide a way to study the properties of solid in the holographic framework $[78,79]$. Also an insulating ground state can be obtained in this way [80]. In addition, another mechanism of momentum dissipation in holographic framework is the Q-lattice [30,31], by which various type of holographic MIT model have been built $[30,31,65,83]$. It is certainly interesting and valuable to incorporate Q-lattice responsible for the momentum dissipation into the Maxwell-Weyl system and study its transport behavior, in particular to see how universal our results are.

3. Another important transport quantity is the magnetotransport, which has been studied with the momentum dissipation[84-87]. We can also study the magnetotransport property in our framework and explore the meaning of EM duality.

4. We would like to study the holographic superconductor in our present framework. In [88], the holographic superconductor with Weyl term is constructed. A main result is that the ratio of the gap frequency over the superconducting critical temperature $\omega_{g} / T_{c}$ runs with the Weyl parameter $\gamma$. Subsequently, a series of works study such holographic superconducting systems with Weyl term, see for example [89-96]. It would be interesting and useful to study the holographic superconducting systems without self-duality but with the momentum dissipation, and further reveal the role that the momentum dissipation plays in the Maxwell-Weyl system.

5. A challenging question is to obtain a full backreaction solution for the EMA-Weyl system. As pointed out in $[17,59]$, we need to develop new numerical technics to solve differential equations beyond the second order with high nonlinearity.

6. In this paper, we consider a four-derivative theory of $g_{\mu \nu}$ and $A_{\mu}$ while the action of the axionic field is only two-derivative. From the viewpoint of an effective field theory, we can also add the four-derivative terms including axionic field, such as $\operatorname{Tr}\left[\Phi^{2}\right] \equiv \Phi_{\nu}^{\mu} \Phi_{\mu}^{\nu}$, and $\operatorname{Tr}\left[\Phi F^{2}\right] \equiv \Phi_{\nu_{1}}^{\mu} F_{\nu_{2}}^{\nu_{1}} F_{\mu}^{\nu_{2}}$ with $\Phi_{\nu}^{\mu}=\frac{1}{2} \sum_{I=x, y} \partial^{\mu} \phi_{I}$ $\partial_{\nu} \phi_{I}$ into the system and study the combined effect on the transports, which have been partially explored in $[80,100]$. Note that when we consider the sixderivative theory, we can add the terms like $C^{2} F^{2}$, $\bar{\Phi} C_{\mu \nu}{ }^{\rho \sigma} F^{\mu \nu} F^{\rho \sigma}, \ldots$, with $\bar{\Phi} \equiv \operatorname{Tr}[\Phi] \equiv \Phi_{\mu}^{\mu}$.

We plan to explore these questions and publish our results in the near future.

Acknowledgements We are very grateful to R. C. Myers, and W. Witczak-Krempa for comments on the QCP. We are also very grateful to Peng Liu, A. Lucas and W. Witczak-Krempa for many useful discussions and comments on the manuscript. We are also grateful to Yi Ling, Peng Liu and Zhenhua Zhou for the collaboration in the related projects. This work is supported by the Natural Science Foundation of China under Grant Nos. 11775036, 11705161 and 11305018. J. P. Wu is also supported by Natural Science Foundation of Liaoning Province under Grant No. 201602013. X. M. Kuang is also supported by Natural Science Foundation of Jiangsu Province under Grant No. BK20170481.

Open Access This article is distributed under the terms of the Creative Commons Attribution 4.0 International License (http://creativecomm ons.org/licenses/by/4.0/), which permits unrestricted use, distribution, and reproduction in any medium, provided you give appropriate credit to the original author(s) and the source, provide a link to the Creative Commons license, and indicate if changes were made.

Funded by SCOAP ${ }^{3}$.

\section{Appendix A: Bounds on the coupling}

The authors of [17,47] examined the causality of the boundary CFT dual to the 4-dimensional SS-AdS geometry and the instabilities of the vector modes in this holographic model. They found that a constraint should be imposed on the coupling $\gamma$ as $\gamma \in \mathcal{S}_{0}$ with $\mathcal{S}_{0}:=[-1 / 12,1 / 12]$. In this appendix, we examine the constraint on $\gamma$ in our present holographic model following [17,47].

It is convenient to write the tensor $X_{\mu \nu}^{\rho \sigma}$ as [17]

$X_{A}^{B}=\left\{X_{1}(u), X_{2}(u), X_{3}(u), X_{4}(u), X_{5}(u), X_{6}(u)\right\}$, 
with

$$
A, B \in\{t x, t y, t u, x y, x u, y u\} .
$$

When the background is rotationally symmetric in $x y$-plane, one has $X_{1}(u)=X_{2}(u)$ and $X_{5}(u)=X_{6}(u)$. Note that from Eq. (8), one can easily find that $\widehat{X}_{A}^{B}$ is also diagonal and its entries $\widehat{X}_{i}$ is the inverse of $X_{i}$, i.e., $\widehat{X}_{i}=1 / X_{i}$.

Turning on the perturbations of the gauge field (19), we obtain the perturbative equations in the planar black brane geometry (14)

$$
\begin{aligned}
& A_{t}^{\prime}+\frac{\hat{q} f}{\hat{\omega}} \frac{X_{5}}{X_{3}} A_{x}^{\prime}=0, \\
& A_{t}^{\prime \prime}+\frac{X_{3}^{\prime}}{X_{3}} A_{t}^{\prime}-\frac{\mathfrak{p}^{2} \hat{q}}{f} \frac{X_{1}}{X_{3}}\left(\hat{q} A_{t}+\hat{\omega} A_{x}\right)=0, \\
& A_{x}^{\prime \prime}+\left(\frac{f^{\prime}}{f}+\frac{X_{5}^{\prime}}{X_{5}}\right) A_{x}^{\prime}+\frac{\mathfrak{p}^{2} \hat{\omega}}{f^{2}} \frac{X_{1}}{X_{5}}\left(\hat{q} A_{t}+\hat{\omega} A_{x}\right)=0, \\
& A_{y}^{\prime \prime}+\left(\frac{f^{\prime}}{f}+\frac{X_{6}^{\prime}}{X_{6}}\right) A_{y}^{\prime}+\frac{\mathfrak{p}^{2}}{f^{2}}\left(\hat{\omega}^{2} \frac{X_{2}}{X_{6}}\right. \\
& \left.\quad-\hat{q}^{2} f \frac{X_{4}}{X_{6}}\right) A_{y}=0,
\end{aligned}
$$

where the prime denotes the derivative with respect to $u$. And then combining Eqs. (A3) and (A4), we can have a decoupled equation of motion for $A_{t}(u, \hat{\mathbf{q}})$ as

$A_{t}^{\prime \prime \prime}+\left(\frac{f^{\prime}}{f}-\frac{X_{1}^{\prime}}{X_{1}}+2 \frac{X_{3}^{\prime}}{X_{3}}\right) A_{t}^{\prime \prime}+\left(-\frac{\mathfrak{p}^{2} \hat{q}^{2} X_{1}}{f X_{3}}+\frac{\mathfrak{p}^{2} \hat{\omega}^{2} X_{1}}{f^{2} X_{5}}\right.$
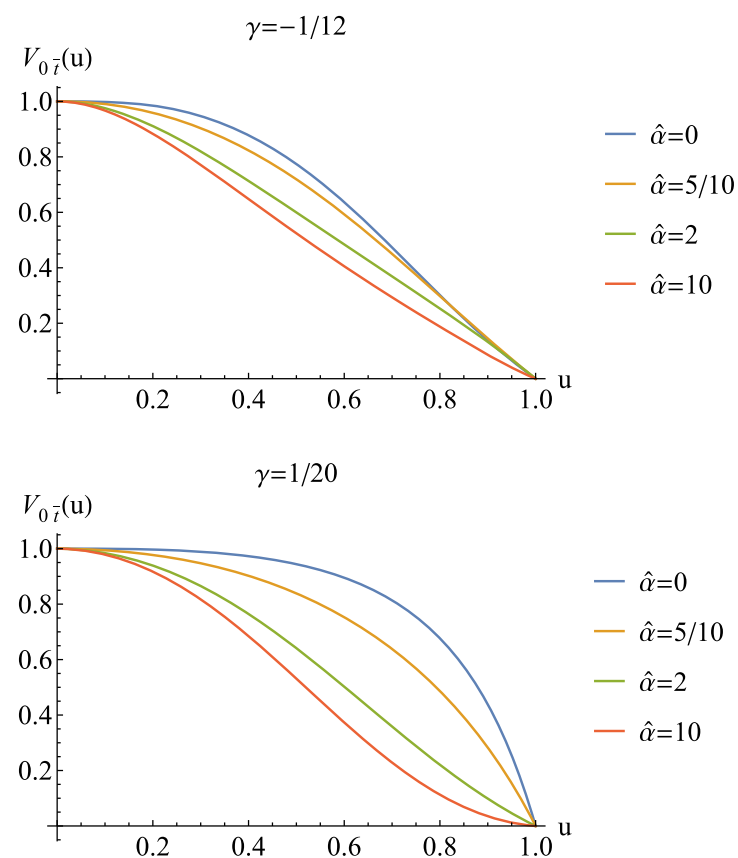

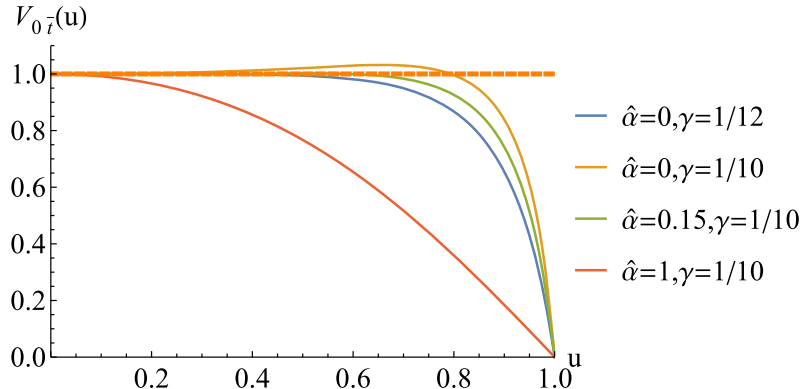

Fig. 14 The shape of the potentials $V_{0 \bar{t}}$ of the longitudinal mode $A_{t}$ with momentum dissipation $(\hat{\alpha} \neq 0)$ and without momentum dissipation $(\hat{\alpha}=0)$ is shown for the $\gamma$ beyond $\mathcal{S}_{0}$. For comparison, $V_{0 \bar{t}}$ with $\hat{\alpha}=0$ and $\gamma=1 / 12$ is also plotted. It clearly show that when the momentum dissipation is introduced, the constraint (A13) can also satisfied for wider region of $\gamma$ beyond $\mathcal{S}_{0}$. (For interpretation of the references to color in this figure, the reader is referred to the web version of this paper)

$$
\left.+\frac{f^{\prime} X_{3}^{\prime}}{f X_{3}}-\frac{X_{1}^{\prime} X_{3}^{\prime}}{X_{1} X_{3}}+\frac{X_{3}^{\prime \prime}}{X_{3}}\right) A_{t}^{\prime}=0
$$

For the dual EM theory, the equations of motion can be obtained by setting $A_{\mu} \rightarrow B_{\mu}$ and $X_{i} \rightarrow \widehat{X}_{i}$ in the above equations.

Next we discuss the bound of $\gamma$ imposed by the causality and the instabilities. Note that since Eq. (A3) gives the relation between $A_{x}^{\prime}$ and $A_{t}^{\prime}$, there are only two independent vector modes $A_{t}$ and $A_{y}$ and we only need to consider the corresponding Eqs. (A6) and (A7). It is convenient to formulate Eqs. (A6) and (A7) into the Schrödinger form. To this end,
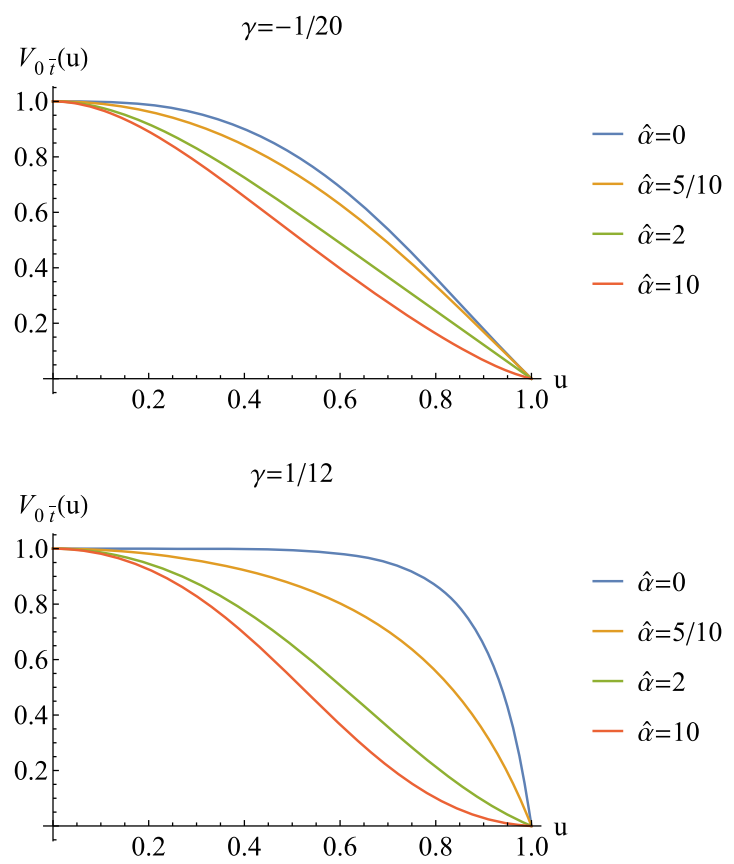

Fig. 13 The shape of the potentials $V_{0 \bar{t}}$ of the longitudinal mode $A_{t}$ for various value of $\gamma \in \mathcal{S}_{0}$ and $\hat{\alpha}$ is shown. We find that $V_{0 \bar{t}}$ well belongs to the region (A13). (For interpretation of the references to color in this figure, the reader is referred to the web version of this paper) 
we make the change of variables $d z / d u=\mathfrak{p} / f$ and write $A_{i}(u)=G_{i}(u) \psi_{i}(u)$ where we denote $A_{\bar{t}}(u):=A_{t}^{\prime}(u)$ and $i=\bar{t}, y$. And then we have

$$
-\partial_{z}^{2} \psi_{i}(z)+V_{i}(u) \psi_{i}(z)=\hat{\omega}^{2} \psi_{i}(z)
$$

where $V_{i}(u)$ is the effective potential. We decompose it into the momentum dependent part and the independent one

$V_{i}(u)=\hat{q}^{2} V_{0 i}(u)+V_{1 i}(u)$,

where [21]

$$
\begin{aligned}
V_{0 \bar{t}} & =f \frac{X_{1}}{X_{3}}, \quad V_{0 y}=f \frac{X_{3}}{X_{1}}, \\
V_{1 \bar{t}} & =\frac{f}{4 \mathfrak{p}^{2} X_{1}^{2}}\left[3 f\left(X_{1}^{\prime}\right)^{2}-2 X_{1}\left(f X_{1}^{\prime}\right)^{\prime}\right], \\
V_{1 y} & =\frac{f}{4 \mathfrak{p}^{2} X_{1}^{2}}\left[-f\left(X_{1}^{\prime}\right)^{2}+2 X_{1}\left(f X_{1}^{\prime}\right)^{\prime}\right] .
\end{aligned}
$$

There is a simple relation between $V_{\bar{t}}$ and $V_{y}$ as $V_{\bar{t}}=$ $\left.V_{y}\right|_{X_{i} \rightarrow \widehat{X}_{i}}$ and vice-versa [21]. At the same time, from Eq. (11), one has $\left.\widehat{X}_{i} \approx X_{i}\right|_{\gamma \rightarrow-\gamma}$ for small $\gamma$. Therefore, we mainly focus on the discussion of $V_{\bar{t}}$ in what follows.

Subsequently, we mainly examine whether the constraint $\gamma \in \mathcal{S}_{0}$ in SS-AdS geometry holds when the momentum dissipation is introduced. For the $\gamma$ beyond $\mathcal{S}_{0}$, we present brief comments. First and foremost we consider the case of the limit of large momentum $(\hat{q} \rightarrow \infty)$. In this limit, the constraint on $V_{0 i}$ should be imposed as

$0 \leq V_{0 i}(u) \leq 1$

The upper bound of $V_{0 i}(u)$ comes from the constraint of the causality in the dual boundary theory [97,98], which is a key constraint on the coupling $\gamma$. Otherwise, there will be super-luminal modes with $\omega / q>1$ in this neutral plasma. While the lower bound of $V_{0 i}(u)$ is from the requirement of stability of the vector modes since in the WKB limit, a negative potential will result in bound states with a negative effective energy, which corresponds to unstable quasinormal modes in the bulk theory [99]. Figure 13 shows the shape of the potential $V_{0 \bar{t}}$ of the longitudinal mode $A_{t}$ for various value of $\gamma \in \mathcal{S}_{0}$ and $\hat{\alpha}$. It implies that $V_{0 \bar{t}}$ well belongs to the region (A13). The similar result is found for the potential $V_{0 y}$ of the transverse mode $A_{y}$. Further careful examination indicates that provided $\gamma \in \mathcal{S}_{0}$ the constraint (A13) is well satisfied for arbitrary $\hat{\alpha}$. In fact, when the momentum dissipation is introduced, the constraint (A13) can be satisfied for wider region of $\gamma$ beyond $\mathcal{S}_{0}$ (see Fig. 14).

Second, we consider the case in the small momentum region, in which $V_{1 i}$ play an important role in the effective potential $V_{i}$. We find that although $V_{1 i}$ develop a negative minimum (see Fig. 15), there are no unstable modes in these regions by analyzing the zero energy bound state in the potential $V_{1 i}$. We shall demonstrate it soon. As analyzed in [99],
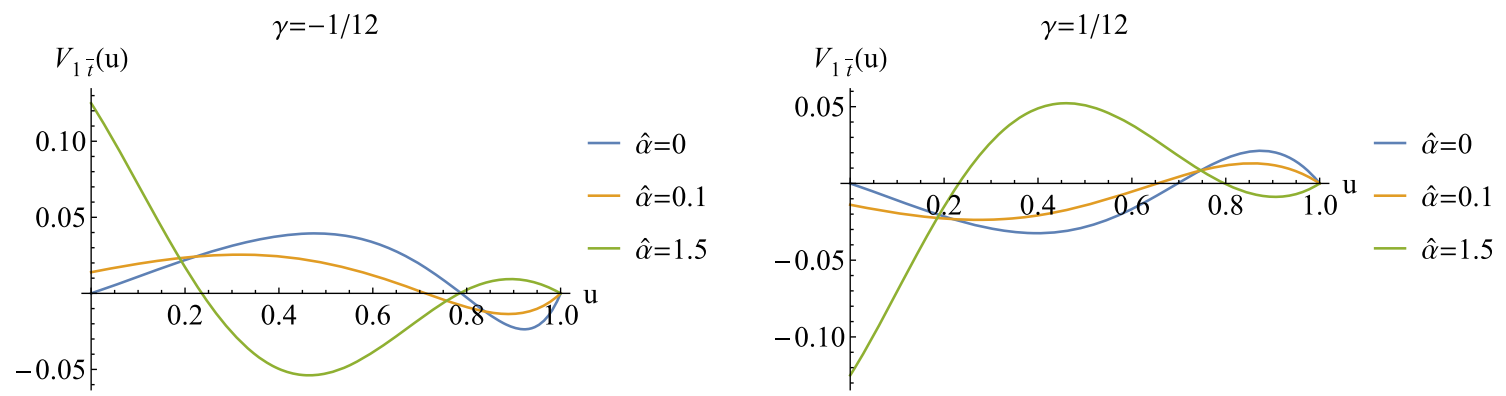

Fig. 15 The shape of the potentials $V_{1 \bar{t}}$ with $\gamma=-1 / 12$ (left plots) and $\gamma=1 / 12$ (right plots) for various value of $\hat{\alpha}$ is shown. (For interpretation of the references to color in this figure, the reader is referred to the web version of this paper)
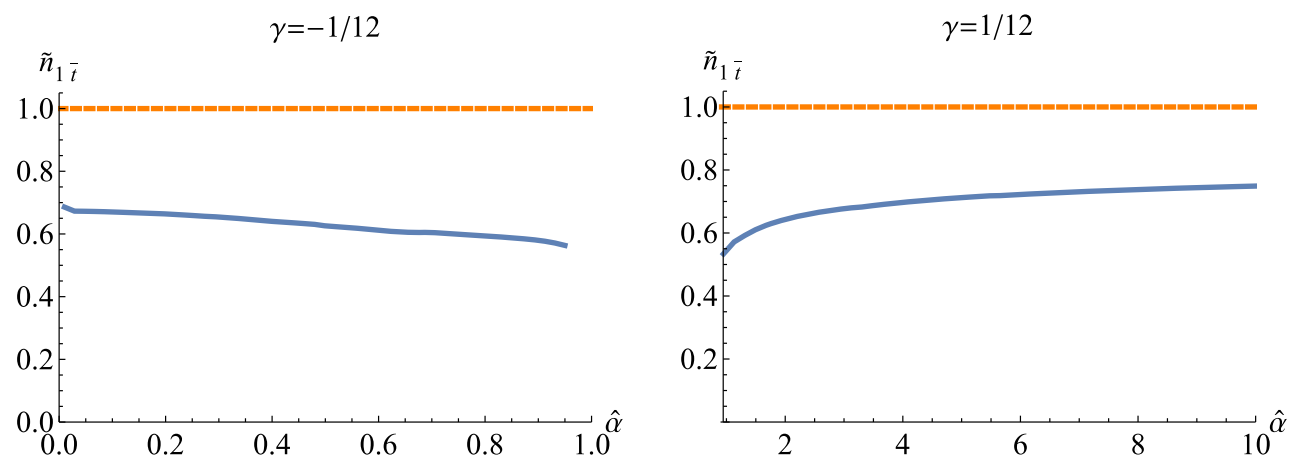

Fig. $16 \tilde{n}_{1 \bar{t}}$ as the function $\hat{\alpha}$ for given $\gamma$ in the region of $\hat{\alpha}$ in which a negative potential develops close to horizon. These plots clearly exhibit that $\tilde{n}_{1 \bar{t}}$ is always less than unit. (For interpretation of the references to color in this figure, the reader is referred to the web version of this paper) 

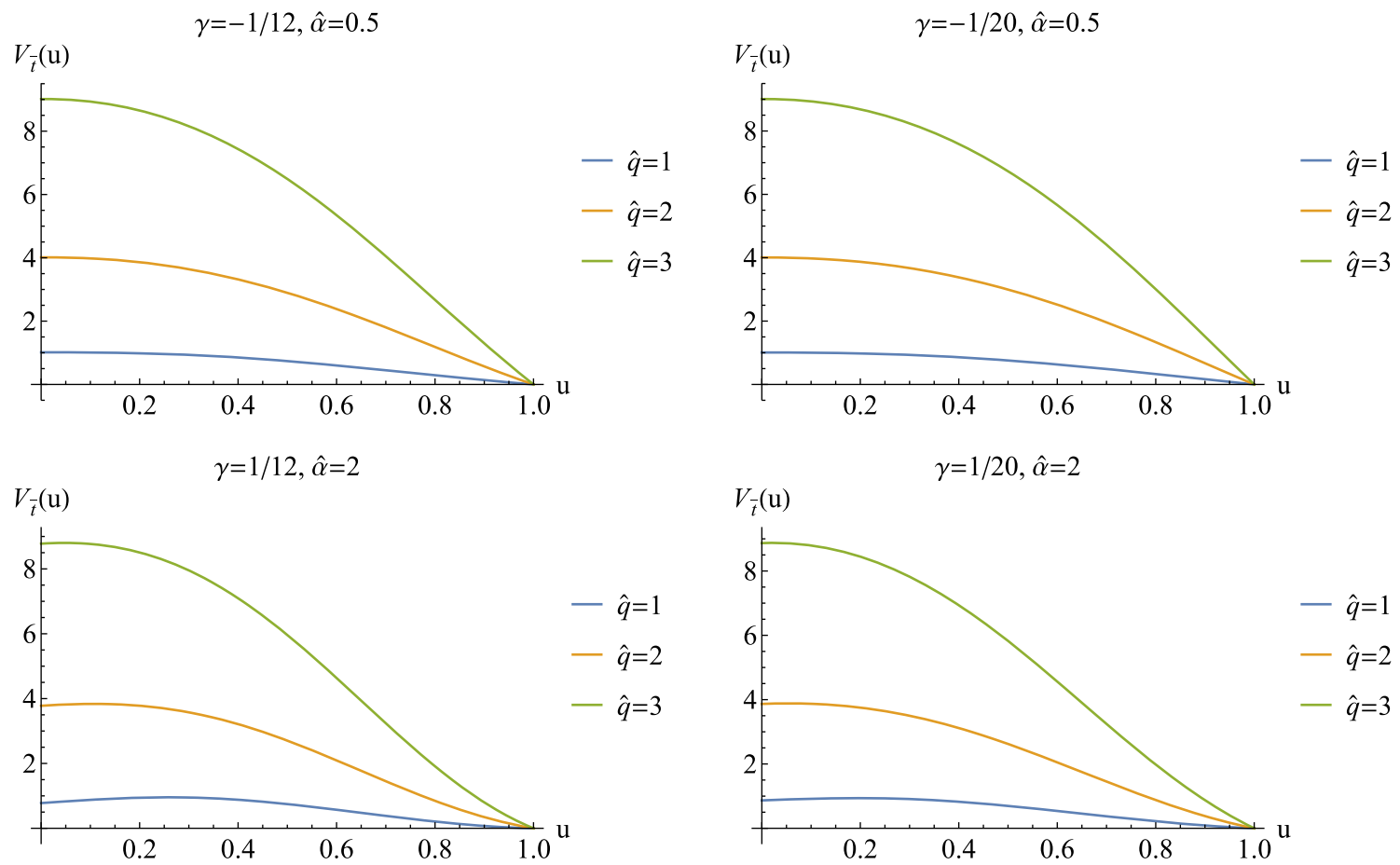

Fig. 17 The potentials $V_{\bar{t}}(u)$ with different $\gamma$ and $\hat{\alpha}$ at some finite momentum. (For interpretation of the references to color in this figure, the reader is referred to the web version of this paper)

there is a zero energy bound state in the potential $V_{1 i}$ by the WKB approximation,

$$
\left(n-\frac{1}{2}\right) \pi=\int_{u_{0}}^{u_{1}} \frac{\mathfrak{p}}{f(u)} \sqrt{-V_{1 i}(u)} d u
$$

where $n$ is a positive integer. The integration is over the values of $u$ for which the potential well is negative. Defining $I_{i} \equiv$ $(n-1 / 2) \pi$ and introducing $\tilde{n}_{i \bar{t}}=I_{i} / \pi+1 / 2$, we plot $\tilde{n}_{1 \bar{t}}$ as the function $\hat{\alpha}$ for given $\gamma$ (see Fig. 16). From this figure, we find that $\tilde{n}_{1 \bar{t}}$ is always less than unit and so no unstable modes appear.

Finally, after examining the instabilities for small and large momentum limit, we examine the instabilities for some finite momentum. Figure 17 shows the potentials $V_{\bar{t}}(u)$ with different $\gamma$ and $\hat{\alpha}$ at some finite momentums. We see that the potential is always positive, which indicates that no unstable modes appear even for the finite momentum. It is because the positive contribution of $V_{0 \bar{t}}(u)$ is larger than the negative one of $V_{1 \bar{t}}(u)$.

We conclude that the region $\gamma \in \mathcal{S}_{0}$ is still physically viable even we introduce in the momentum dissipation. In fact, this physically viable region may become larger in this neutral axionic geometry (14) (see for example Fig. 14). More detailed exploration will be left for the future and here we only restrict our studies in the region $\gamma \in \mathcal{S}_{0}$.

\section{References}

1. K. Damle, S. Sachdev, Nonzero-temperature transport near quantum critical points. Phys. Rev. B 56(14), 8714 (1997). arXiv:cond-mat/9705206 [cond-mat.str-el]

2. S. Sachdev, Nonzero temperature transport near fractional quantum Hall critical points. Phys. Rev. B 57, 7157 (1998). arXiv:cond-mat/9709243 [cond-mat.mes-hall]

3. L. Fritz, J. Schmalian, M. Mueller, S. Sachdev, Quantum critical transport in clean graphene. Phys. Rev. B 78, 085416 (2008). arXiv:0802.4289 [cond-mat.mes-hall]

4. W. Witczak-Krempa, P. Ghaemi, T. Senthil, Y.B. Kim, Universal transport near a quantum critical Mott transition in two dimensions. Phys. Rev. B 86, 245102 (2012). arXiv:1206.3309 [condmat.str-el]

5. J.M. Maldacena, The large N limit of superconformal field theories and supergravity. Int. J. Theor. Phys. 38, 1113 (1999)

6. J.M. Maldacena, Adv. Theor. Math. Phys. 2, 231 (1998). arXiv:hep-th/9711200

7. S.S. Gubser, I.R. Klebanov, A.M. Polyakov, Gauge theory correlators from noncritical string theory. Phys. Lett. B 428, 105 (1998). arXiv:hep-th/9802109

8. E. Witten, Anti-de Sitter space and holography. Adv. Theor. Math. Phys. 2, 253 (1998). arXiv:hep-th/9802150

9. O. Aharony, S.S. Gubser, J.M. Maldacena, H. Ooguri, Y. Oz, Large $\mathrm{N}$ field theories, string theory and gravity. Phys. Rep. 323, 183 (2000). arXiv:hep-th/9905111

10. S. Sachdev, Quantum Phase Transitions, 2nd edn. (Cambridge University Press, England, 2011)

11. Min-Chul Cha, Matthew P.A. Fisher, S.M. Girvin, Mats Wallin, A.Peter Young, Universal conductivity of two-dimensional films at the superconductor-insulator transition. Phys. Rev. B 44, 68836902 (1991) 
12. J. Smakov, E. Sorensen, Universal scaling of the conductivity at the superfluid-insulator phase transition. Phys. Rev. Lett. 95, 180603 (2005). arXiv:cond-mat/0509671

13. K. Chen, L. Liu, Y. Deng, L. Pollet, N. Prokof'ev, Universal conductivity in a two-dimensional superfluid-to-insulator quantum critical system. Phys. Rev. Lett. 112(3), 030402 (2014). arXiv:1309.5635 [cond-mat.str-el]

14. S. Gazit, D. Podolsky, A. Auerbach, D.P. Arovas, Dynamics and conductivity near quantum criticality. Phys. Rev. B 88, 235108 (2013). arXiv:1309.1765 [cond-mat.str-el]

15. S. Gazit, D. Podolsky, A. Auerbach, Critical capacitance and charge-vortex duality near the superfluid-to-insulator transition. Phys. Rev. Lett. 113, 240601 (2014). arXiv:1407.1055 [condmat.str-el]

16. W. Witczak-Krempa, J. Maciejko, Optical conductivity of topological surface states with emergent supersymmetry. Phys. Rev. Lett. 116(10), 100402 (2016). arXiv:1510.06397 [cond-mat.strel]

17. R.C. Myers, S. Sachdev, A. Singh, Holographic quantum critical transport without self-duality. Phys. Rev. D 83, 066017 (2011). arXiv: 1010.0443 [hep-th]

18. W. Witczak-Krempa, S. Sachdev, The quasi-normal modes of quantum criticality. Phys. Rev. B 86, 235115 (2012). arXiv:1210.4166 [cond-mat.str-el]

19. W. Witczak-Krempa, S. Sachdev, Dispersing quasinormal modes in 2+1 dimensional conformal field theories. Phys. Rev. B 87, 155149 (2013). arXiv:1302.0847 [cond-mat.str-el]

20. W. Witczak-Krempa, E.S. Sørensen, S. Sachdev, The dynamics of quantum criticality via quantum Monte Carlo and holography. Nature Phys. 10, 361 (2014). arXiv:1309.2941 [cond-mat.str-el]

21. W. Witczak-Krempa, Quantum critical charge response from higher derivatives in holography. Phys. Rev. B 89(16), 161114 (2014). arXiv:1312.3334 [cond-mat.str-el]

22. E. Katz, S. Sachdev, E.S. Sørensen, W. Witczak-Krempa, Conformal field theories at nonzero temperature: operator product expansions, Monte Carlo, and holography. Phys. Rev. B 90(24), 245109 (2014). arXiv:1409.3841 [cond-mat.str-el]

23. S. Bai, D.W. Pang, Holographic charge transport in $2+1$ dimensions at finite $N$. Int. J. Mod. Phys. A 29, 1450061 (2014). arXiv:1312.3351 [hep-th]

24. C.P. Herzog, P. Kovtun, S. Sachdev, D.T. Son, Quantum critical transport, duality, and M-theory. Phys. Rev. D 75, 085020 (2007). arXiv:hep-th/0701036

25. S. Sachdev, What can gauge-gravity duality teach us about condensed matter physics? Ann. Rev. Condens. Matter Phys. 3, 9 (2012). arXiv:1108.1197 [cond-mat.str-el]

26. G.T. Horowitz, J.E. Santos, D. Tong, Optical conductivity with holographic lattices. JHEP 1207, 168 (2012). arXiv:1204.0519 [hep-th]

27. G.T. Horowitz, J.E. Santos, D. Tong, Further evidence for latticeinduced scaling. JHEP 1211, 102 (2012). arXiv:1209.1098 [hepth]

28. Y. Ling, C. Niu, J.P. Wu, Z.Y. Xian, Holographic lattice in Einstein-Maxwell-Dilaton gravity. JHEP 1311, 006 (2013). arXiv: 1309.4580 [hep-th]

29. A. Donos, S.A. Hartnoll, Interaction-driven localization in holography. Nat. Phys. 9, 649 (2013). arXiv:1212.2998

30. A. Donos, J.P. Gauntlett, Holographic Q-lattices. JHEP 1404, 040 (2014). arXiv:1311.3292 [hep-th]

31. A. Donos, J.P. Gauntlett, Novel metals and insulators from holography. JHEP 1406, 007 (2014). arXiv: 1401.5077 [hep-th]

32. D. Vegh, Holography without translational symmetry. arXiv: 1301.0537 [hep-th]

33. T. Andrade, B. Withers, A simple holographic model of momentum relaxation. JHEP 1405, 101 (2014). arXiv:1311.5157 [hep-th]
34. M. Blake, D. Tong, Universal resistivity from holographic massive gravity. Phys. Rev. D 88(10), 106004 (2013). arXiv:1308.4970 [hep-th]

35. M. Blake, D. Tong, D. Vegh, Holographic lattices give the graviton an effective mass. Phys. Rev. Lett. 112(7), 071602 (2014). arXiv: 1310.3832 [hep-th]

36. Y. Ling, P. Liu, J.P. Wu, Characterization of quantum phase transition using holographic entanglement entropy. Phys. Rev. D 93(12), 126004 (2016). arXiv:1604.04857 [hep-th]

37. Y. Ling, P. Liu, C. Niu, J.P. Wu, Z.Y. Xian, Holographic entanglement entropy close to quantum phase transitions. JHEP 1604, 114 (2016). arXiv: 1502.03661 [hep-th]

38. Y. Ling, P. Liu, C. Niu, J.P. Wu, Z.Y. Xian, Holographic superconductor on Q-lattice. JHEP 1502, 059 (2015). arXiv:1410.6761 [hep-th]

39. Y. Ling, C. Niu, J. Wu, Z. Xian, H.b Zhang, Metal-insulator transition by holographic charge density waves. Phys. Rev. Lett. 113, 091602 (2014). arXiv:1404.0777 [hep-th]

40. H .B. Zeng, J .P. Wu, Holographic superconductors from the massive gravity. Phys. Rev. D 90(4), 046001 (2014). arXiv:1404.5321 [hep-th]

41. L.Q. Fang, X.M. Kuang, B. Wang, J.P. Wu, Fermionic phase transition induced by the effective impurity in holography. JHEP 1511, 134 (2015). arXiv:1507.03121 [hep-th]

42. L.Q. Fang, X.M. Kuang, J.P. Wu, The holographic fermions dual to massive gravity. Sci. China Phys. Mech. Astron. 59(10), 100411 (2016)

43. M. Reza Mohammadi Mozaffar, A. Mollabashi, F. Omidi, Nonlocal probes in holographic theories with momentum relaxation. arXiv:1608.08781 [hep-th]

44. X.M. Kuang, E. Papantonopoulos, J.P. Wu, Z. Zhou, The Lifshitz black branes and DC transport coefficients in massive EinsteinMaxwell-Dilaton gravity. arXiv:1709.02976 [hep-th]

45. S. Grozdanov, A. Lucas, S. Sachdev, K. Schalm, Absence of disorder-driven metal-insulator transitions in simple holographic models. Phys. Rev. Lett. 115(22), 221601 (2015). arXiv:1507.00003 [hep-th]

46. A. Lucas, S. Gazit, D. Podolsky, W. Witczak-Krempa, Dynamical response near quantum critical points. arXiv:1608.02586 [condmat.str-el]

47. A. Ritz, J. Ward, Weyl corrections to holographic conductivity. Phys. Rev. D 79, 066003 (2009). arXiv:0811.4195 [hep-th]

48. K. Hanaki, K. Ohashi, Y. Tachikawa, Supersymmetric completion of an $\mathrm{R} * * 2$ term in five-dimensional supergravity. Prog. Theor. Phys. 117, 533 (2007). arXiv:hep-th/0611329

49. S. Cremonini, K. Hanaki, J.T. Liu, P. Szepietowski, Black holes in five-dimensional gauged supergravity with higher derivatives. JHEP 0912, 045 (2009). arXiv:0812.3572 [hep-th]

50. K.Y. Kim, K.K. Kim, Y. Seo, S.J. Sin, Coherent/incoherent metal transition in a holographic model. JHEP 1412, 170 (2014). arXiv:1409.8346 [hep-th]

51. X.M. Kuang, J.P. Wu, Phys. Lett. B 770, 117 (2017). arXiv:1702.01490 [hep-th]

52. R.A. Davison, B. Goutraux, Momentum dissipation and effective theories of coherent and incoherent transport. JHEP 1501, 039 (2015). arXiv:1411.1062 [hep-th]

53. R.C. Myers, M.F. Paulos, A. Sinha, Holographic hydrodynamics with a chemical potential. JHEP 0906, 006 (2009). arXiv:0903.2834 [hep-th]

54. J.T. Liu, P. Szepietowski, Higher derivative corrections to Rcharged AdS(5) black holes and field redefinitions. Phys. Rev. D 79, 084042 (2009). arXiv:0806.1026 [hep-th]

55. R.G. Cai, D.W. Pang, Holography of charged black holes with $R F^{2}$ corrections. Phys. Rev. D 84, 066004 (2011). arXiv:1104.4453 [hep-th] 
56. A. Dey, S. Mahapatra, T. Sarkar, Holographic thermalization with Weyl corrections. JHEP 1601, 088 (2016). arXiv:1510.00232 [hep-th]

57. A. Dey, S. Mahapatra, T. Sarkar, Thermodynamics and entanglement entropy with Weyl corrections. Phys. Rev. D 94(2), 026006 (2016). arXiv: 1512.07117 [hep-th]

58. S. Mahapatra, Thermodynamics, phase transition and quasinormal modes with Weyl corrections. JHEP 1604, 142 (2016). arXiv: 1602.03007 [hep-th]

59. Y. Ling, P. Liu, J.P. Wu, Z. Zhou, Holographic metal-insulator transition in higher derivative gravity. arXiv:1606.07866 [hep-th]

60. S.A. Hartnoll, Theory of universal incoherent metallic transport. Nat. Phys. 11(54) (2015). arXiv:1405.3651 [cond-mat.str-el]

61. X.H. Ge, Y. Ling, C. Niu, S.J. Sin, Thermoelectric conductivities, shear viscosity, and stability in an anisotropic linear axion model. Phys. Rev. D 92(10), 106005 (2015). arXiv:1412.8346 [hep-th]

62. R.A. Davison, B. Gouteraux, S.A. Hartnoll, Incoherent transport in clean quantum critical metals. JHEP 1510, 112 (2015). arXiv: 1507.07137 [hep-th]

63. R.A. Davison, B. Gouteraux, Dissecting holographic conductivities. JHEP 1509, 090 (2015). arXiv: 1505.05092 [hep-th]

64. Z. Zhou, Y. Ling, J.P. Wu, Holographic incoherent transport in Einstein-Maxwell-Dilaton gravity. arXiv:1512.01434 [hep-th]

65. Y. Ling, P. Liu, J.P. Wu, A novel insulator by holographic Qlattices. JHEP 1602, 075 (2016). arXiv: 1510.05456 [hep-th]

66. S.A. Hartnoll, A. Lucas, S. Sachdev, Holographic quantum matter. arXiv:1612.07324 [hep-th]

67. S.A. Hartnoll, D.M. Hofman, Locally critical resistivities from umklapp scattering. Phys. Rev. Lett. 108, 241601 (2012). arXiv:1201.3917 [hep-th]

68. C.F. Chen, A. Lucas, Origin of the Drude peak and of zero sound in probe brane holography. Phys. Lett. B 774, 569 (2017). arXiv: 1709.01520 [hep-th]

69. P. Kovtun, D.T. Son, A.O. Starinets, Holography and hydrodynamics: diffusion on stretched horizons. JHEP 0310, 064 (2003). arXiv:hep-th/0309213

70. M. Brigante, H. Liu, R.C. Myers, S. Shenker, S. Yaida, Viscosity bound violation in higher derivative gravity. Phys. Rev. D 77, 126006 (2008). arXiv:0712.0805 [hep-th]

71. S. Grozdanov, A.O. Starinets, Second-order transport, quasinormal modes and zero-viscosity limit in the Gauss-Bonnet holographic fluid. JHEP 1703, 166 (2017). arXiv: 1611.07053 [hep-th]

72. R.C. Myers, T. Sierens, W. Witczak-Krempa, A holographic model for quantum critical responses. JHEP 1605, 073 (2016). arXiv: 1602.05599 [hep-th]

73. J. Zaanen, Y.-W. Sun, Y. Liu, K. Schalm, Holographic Duality in Condensed Matter Physics (Cambridge Univ. Press, Cambridge, 2015)

74. M. Ammon, J. Erdmenger, Gauge/gravity duality (Cambridge Univ. Press, Cambridge, 2015)

75. B. Keimer, S.A. Kivelson, M.R. Norman, S. Uchida, J. Zaanen, From quantum matter to high-temperature superconductivity in copper oxides. Nature 518(02), 179C186 (2015). arXiv:1409.4673 [cond-mat.supr-con]

76. D. Forcella, J. Zaanen, D. Valentinis, D. van der Marel, Electromagnetic properties of viscous charged fluids. Phys. Rev. B 90(3), 035143 (2014). arXiv:1406.1356 [cond-mat.str-el]

77. S. Vig, A. Kogar, V. Mishra, L. Venema, M. S. Rak, A.A. Husain, P.D. Johnson, G.D. Gu, E. Fradkin, M.R. Norman, P. Abbamonte, Fluctuating charge order in the optimally doped high temperature superconductor $\mathrm{Bi} 2 \mathrm{Sr} 2 \mathrm{CaCu} 2 \mathrm{O} 8+\mathrm{x}$. arXiv:1509.04230 [cond-mat.supr-con]
78. L. Alberte, M. Baggioli, A. Khmelnitsky, O. Pujolas, Solid holography and massive gravity. JHEP 1602, 114 (2016). arXiv:1510.09089 [hep-th]

79. L. Alberte, M. Baggioli, O. Pujolas, Viscosity bound violation in holographic solids and the viscoelastic response. JHEP 1607, 074 (2016). arXiv:1601.03384 [hep-th]

80. B. Gouteraux, E. Kiritsis, W.J. Li, Effective holographic theories of momentum relaxation and violation of conductivity bound. JHEP 1604, 122 (2016). arXiv:1602.01067 [hep-th]

81. M. Baggioli, O. Pujolas, Electron-phonon interactions, metalinsulator transitions, and holographic massive gravity. Phys. Rev. Lett. 114(25), 251602 (2015). arXiv:1411.1003 [hep-th]

82. M. Baggioli, O. Pujolas, On holographic disorder-driven metalinsulator transitions. arXiv:1601.07897 [hep-th]

83. Y. Ling, P. Liu, C. Niu, J.P. Wu, Building a doped Mott system by holography. Phys. Rev. D 92(8), 086003 (2015). arXiv:1507.02514 [hep-th]

84. A. Amoretti, A. Braggio, N. Maggiore, N. Magnoli, D. Musso, Thermo-electric transport in gauge/gravity models with momentum dissipation. JHEP 1409, 160 (2014). arXiv:1406.4134 [hepth]

85. A. Amoretti, A. Braggio, N. Maggiore, N. Magnoli, D. Musso, Analytic dc thermoelectric conductivities in holography with massive gravitons. Phys. Rev. D 91(2), 025002 (2015). arXiv: 1407.0306 [hep-th]

86. A. Amoretti, D. Musso, Magneto-transport from momentum dissipating holography. JHEP 1509, 094 (2015). arXiv:1502.02631 [hep-th]

87. A. Amoretti, M. Baggioli, N. Magnoli, D. Musso, Chasing the cuprates with dilatonic dyons. JHEP 1606, 113 (2016). arXiv: 1603.03029 [hep-th]

88. J.P. Wu, Y. Cao, X.M. Kuang, W.J. Li, The $3+1$ holographic superconductor with Weyl corrections. Phys. Lett. B 697, 153 (2011). arXiv: 1010.1929 [hep-th]

89. D.Z. Ma, Y. Cao, J.P. Wu, The Stuckelberg holographic superconductors with Weyl corrections. Phys. Lett. B 704, 604 (2011). arXiv:1201.2486 [hep-th]

90. D. Momeni, M.R. Setare, A note on holographic superconductors with Weyl corrections. Mod. Phys. Lett. A 26, 2889 (2011). arXiv:1106.0431 [physics.gen-ph]

91. D. Momeni, N. Majd, R. Myrzakulov, p-wave holographic superconductors with Weyl corrections. Europhys. Lett. 97, 61001 (2012). arXiv:1204.1246 [hep-th]

92. Z. Zhao, Q. Pan, J. Jing, Holographic insulator/superconductor phase transition with Weyl corrections. Phys. Lett. B 719, 440 (2013). arXiv:1212.3062

93. D. Momeni, R. Myrzakulov, M. Raza, Holographic superconductors with Weyl corrections via gauge/gravity duality. Int. J. Mod. Phys. A 28, 1350096 (2013). arXiv:1307.8348 [hep-th]

94. D. Momeni, M. Raza, R. Myrzakulov, Holographic superconductors with Weyl corrections. Int. J. Geom. Methods Mod. Phys. 13, 1550131 (2016). arXiv:1410.8379 [hep-th]

95. L. Zhang, Q. Pan, J. Jing, Holographic p-wave superconductor models with Weyl corrections. Phys. Lett. B 743, 104 (2015). arXiv:1502.05635 [hep-th]

96. S.A.H. Mansoori, B. Mirza, A. Mokhtari, F.L. Dezaki, Z. Sherkatghanad, Weyl holographic superconductor in the Lifshitz black hole background. JHEP 1607, 111 (2016). arXiv:1602.07245 [hep-th]

97. A. Buchel, R.C. Myers, Causality of holographic hydrodynamics. JHEP 0908, 016 (2009). arXiv:0906.2922 [hep-th] 
98. M. Brigante, H. Liu, R.C. Myers, S. Shenker, S. Yaida, The viscosity bound and causality violation. Phys. Rev. Lett. 100, 191601 (2008). arXiv:0802.3318 [hep-th]

99. R.C. Myers, A.O. Starinets, R.M. Thomson, Holographic spectral functions and diffusion constants for fundamental matter. JHEP 0711, 091 (2007). arXiv:0706.0162 [hep-th]
100. M. Baggioli, B. Goutéraux, E. Kiritsis, W.J. Li, Higher derivative corrections to incoherent metallic transport in holography. JHEP 1703, 170 (2017). arXiv:1612.05500 [hep-th] 